\title{
Organocatalysis in heterocyclic synthesis: DABCO as a mild and efficient catalytic system for the synthesis of a novel class of quinazoline, thiazolo [3,2-a]quinazoline and thiazolo[2,3-b] quinazoline derivatives
}

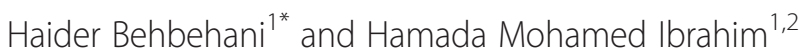

\begin{abstract}
Background: There are only limited publications devoted to the synthesis of especially thiazolo[3,2-a]quinazoline which involved reaction of 2-mercaptopropargyl quinazolin-4-one with various aryl iodides catalyzed by Pd-Cu or by condensation of 2-mercapto-4-oxoquinazoline with chloroacetic acid, inspite of this procedure was also reported in the literature to afford the thiazolo [2,3-b] quinazoline. So the multistep synthesis of the thiazolo[3,2-a]quinazoline suffered from some flaws and in this study we have synthesized a novel class of thiazoloquinazolines by a simple and convenient method involving catalysis by 1,4-diazabicyclo[2.2.2]octane (DABCO).

Results: A new and convenient one-pot synthesis of a novel class of 2-arylidene-2H-thiazolo[3,2-a]quinazoline-1, 5-diones 9a-i was established through the reaction between methyl-2-(2-thio-cyanatoacetamido)benzoate (4) and a variety of arylidene malononitriles 8a-i in the presence of DABCO as a mild and efficient catalytic system via a Michael type addition reaction and a mechanism for formation of the products observed is proposed. Moreover 4 was converted to ethyl-2-[(4-oxo-3,4-dihydroquinazolin-2-yl)thio]acetate (10) upon reflux in ethanol containing $\mathrm{DABCO}$ as catalyst. The latter was reacted with aromatic aldehydes and dimethylformamide dimethylacetal (DMF-DMA) to afford a mixture of two regioselectively products with identical percentage yield, these two products were identified as thiazolo[3,2-a]quinazoline 9,13 and thiazolo[2,3-b]quinazoline 11,12 derivatives respectively. The structure of the compounds prepared in this study was elucidated by different spectroscopic tools of analyses also the X-ray single crystal technique was employed in this study for structure elucidation, Z/E potential isomerism configuration determination and to determine the regioselectivity of the reactions.

Conclusion: A simple and efficient one-pot synthesis of a novel class of 2-arylidene-2H-thiazolo[3,2-a]quinazoline-1, 5-diones 9a-i was established through DABCO catalyzed Michael type addition reaction. In addition many fused quinazoline and quinazoline derivatives were synthesized which appeared as valuable precursors in synthetic and medicinal chemistry.
\end{abstract}

Keywords: Organocatalysis, DABCO, Quinazoline, Thiazolo[3,2-a] quinazoline, Thiazolo[2,3-b]quinazoline

\footnotetext{
* Correspondence: hidar@bahbahani.com

${ }^{1}$ Chemistry Department, Faculty of Science, Kuwait University, P.O. Box 5969,

Safat 13060, Kuwait

Full list of author information is available at the end of the article
} 


\section{Background}

The synthesis of fused heterocycles has attracted considerable interest in heterocyclic chemistry as the fusion of biodynamic heterosystems has proved to be a very attractive and useful for the design of new molecular framework of potential drugs with varying pharmacological activities. A major challenge of the modern synthetic chemistry is to design highly efficient chemical reaction sequences which provide molecules containing maximum complexity and structural diversity with interesting bioactivities in minimum number of synthetic steps. Recently, organocatalysis has increased spectacularly in the last few years as a result of both the novelty of the concept and, more importantly, the fact that the efficiency and the selectivity of many organocatalytic reactions meet the standards of established organic reactions. One of these organocatalysts is the 1 , 4-diazabicyclo[2.2.2] octane (DABCO) which has received considerable attention as an inexpensive, eco-friendly, high reactive and non-toxic base catalyst for various organic transformations, affording the corresponding products in excellent yields with high selectivity [1-5]. We have found that the quinazolines and condensed quinazolines are versatile classes of fused heterocycles that are of considerable interest because of the diverse range of their biological properties and the potent pharmacological activities such as anticancer [6,7], antitumor [8,9], antioxidant [10], analgesic [11], anti-inflammatory [7,12], anticonvulsant [13], anti HIV, antibacterial, antifungal [14-17], antihypertensive [18], antileishmanial [19] and CNS depressant activity [20]. On the other hand, the considerable biological and medicinal activities of the thiazoles and their derivatives have also attracted continuing interest over the years because of their varied biological activities exemplified as antibacterial, antifungal [21-24], antitubercular [25], anticancer [26-28], antidiabetic [29], anti HIV [30] in addition to large applications in the drug development for the treatment of many disease. So, on the basis of the above findings the quinazoline and thiazole are privileged structures, which attracted considerable attention in the designing of biologically active molecules and combining them in one molecule exemplified by the thiazoloquinazoline system it is expected to furnish biologically active molecule with characteristic features. In the last decade numerous methods have been developed for the synthesis of highly substituted thiazoloquinazoline system exemplified by thiazolo[2,3- $b$ ] quinazoline [8,31-33], thiazolo[5,4-f]quinazoline [34,35], thiazolo[4,5h]quinazolin [36], thiazolo[5,4-c]quinoline [37], thiazolo $[4,3-b]$ quinazoline [38] and thiazolo[3,2-a]quinazoline [39]. However, after detailed literature survey it was observed that there were only limited publications devoted to the synthesis of especially thiazolo[3,2-a]quinazoline which involved reaction of 2-mercaptopropargyl quinazolin-4-one with various aryl iodides catalyzed by
$\mathrm{Pd}-\mathrm{Cu}$ [39] or by condensation of 2-mercapto-4oxoquinazoline with chloroacetic acid [40], inspite of this procedure was also reported in the literature to afford the thiazolo[2,3-b]quinazoline. So the multistep synthesis of thiazoloquinazolines especially thiazolo[3,2-a]quinazoline suffered from some flaws and in continuation of our research program on the synthesis of nitrogen and sulphur containing novel heterocycles [41-43] of pharmaceutical interest and in view of the operational simplicity in this study we have synthesized a novel class of thiazoloquinazolines by a simple and convenient method involving catalysis by $\mathrm{DABCO}$. The $\mathrm{X}$-ray single crystal technique as an advanced tool of analysis was employed in this study for structure elucidation and for determination the regioselectivety of the reactions.

\section{Results and discussion}

\section{Synthetic chemistry}

The synthetic strategy of our study to obtain the targeted compounds began by preparing the starting material methyl-2-(2-thiocyanatoacetamido)benzoate (4) which prepared in two synthetic steps firstly by reacting the methyl anthranilate (MA) (1) with chloroacetyl chloride (2) to afford methyl-2-(2-chloroacetamido)benzoate (3) and secondly by reacting the latter with ammonium thiocyanate in refluxing acetone. Moreover conducting the reaction between the methyl-2-(2-chloroaceta-mido) benzoate (3) and the ammonium thiocyanate in absolute methanol afford two products depending on the refluxing time, 4 was formed after $6 \mathrm{~h}$ while the methyl-2-[(4-oxo3,4-dihydroquinazolin-2-yl)thio]acetate (5) was formed after $12 \mathrm{~h}$ and not compound $\mathbf{6}$ or 7 [43,44]. Also compound 5 can be obtained by refluxing the formed methyl2-(2-thiocyanatoacetamido)benzoate (4) in methanol [45] (cf. Schemes 1 and 2). The structure of compounds 3, 4 and 5 was confirmed through the X-ray single crystal structure determination (cf. Figures 1, 2, 3).

Now it was of interest to explore the scope and limitations and generality of the methyl-2-(2-thiocyanatoacetamido)benzoate (4) as a precursor for the synthesis of some polyfunctionally substituted fused thiazoloquinazoline derivatives for which we might expect a wide spectrum of bioresponses. Thus the active methylene in the methyl2-(2-thiocyanatoacetamido)benzoate (4) underwent nucleophilic addition reaction to the double bond of a variety of arylidene malononitriles 8a-i via a Michael type addition reaction [42], by refluxing in ethanol containing $10 \mathrm{~mol} \%$ of $\mathrm{DABCO}$ as catalyst to give a substance whose structure was determined as thiazolo[3,2-a]quinazoline derivatives 9a-i, as established from the accurate mass determination, ${ }^{1} \mathrm{H}-\mathrm{NMR}$ and ${ }^{13} \mathrm{C}$-NMR. Moreover this structure was also confirmed through the X-ray single crystal structure determination for 9a, 9c, 9d, 9f and $\mathbf{9 h}$ (cf. Figures 4, 5, 6, 7, 8 and Scheme 3). It is worth mention that the short reaction 


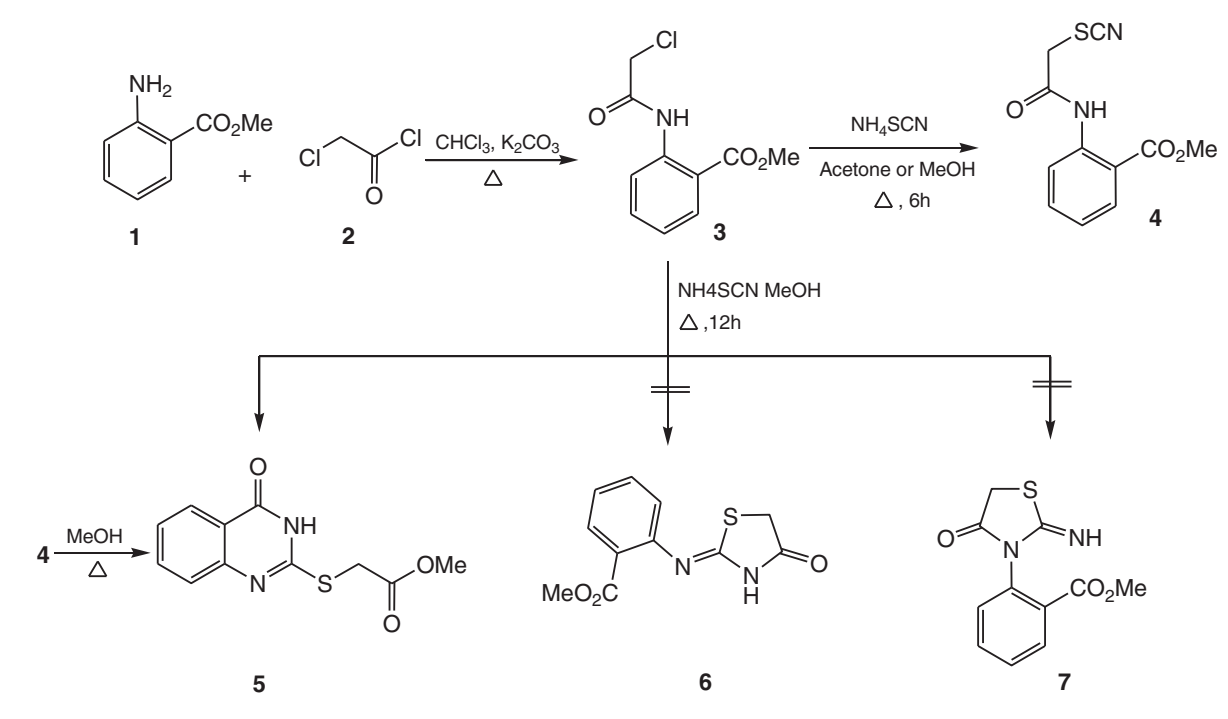

Scheme 1 Synthesis of methyl-2-(2-thiocyanatoacetamido)benzoate (4) and the quinazoline derivative 5.

times, easy workup, very good to excellent yields, and mild reaction conditions make this Michael type addition reaction followed by interamolecular cyclization both practical and attractive. It is believed that initially the carbanion which formed from 4 by the action of the base (B:) DABCO undergoes nucleophilic addition to the double bond of the arylidene malononitrile to form the adduct $\mathbf{B}$ followed by losing one molecule of malononitrile and subsequent interamolecular cyclization forming the thiazolidinone ring via attack of the $\mathrm{NH}$ moiety at $\mathrm{SCN}$, and finally the thiazolo[3,2-a]quinazoline derivatives 9 was formed through losing one molecule of methanol during the another interamolecular cyclization. Also in order to confirm the above synthetic route we try to separate one of the formed intermediates during the reaction and we success to isolate the intermediate $\mathbf{D}\left[\mathrm{Ar}=P-\mathrm{NO}_{2} \mathrm{C}_{6} \mathrm{H}_{4}\right]$ during the preparation of 9e. The X-ray single crystal technique was successfully employed in this study to confirm the potential formation of the $\mathrm{Z}$ isomer during the preparation of $\mathbf{9}$ as a sole isolable isomer product.

To optimize the reaction conditions, we systematically investigated the reaction parameters using 4 and benzylidene malononitriles $\mathbf{8 a}$ (Table 1). First, the effect of bases was investigated (entries 1-6). It was found that<smiles>COC(=O)c1ccccc1NC(=O)CCl</smiles>

3<smiles>COC(=O)c1ccccc1NC(=O)CSC#N</smiles>

4<smiles>COc1ccccc1N1C(=N)SCC1C</smiles>
$-\mathrm{MeOH}$ $\mathrm{ROH}$<smiles>O=C1CSc2nc(=O)c3ccccc3n21</smiles> 


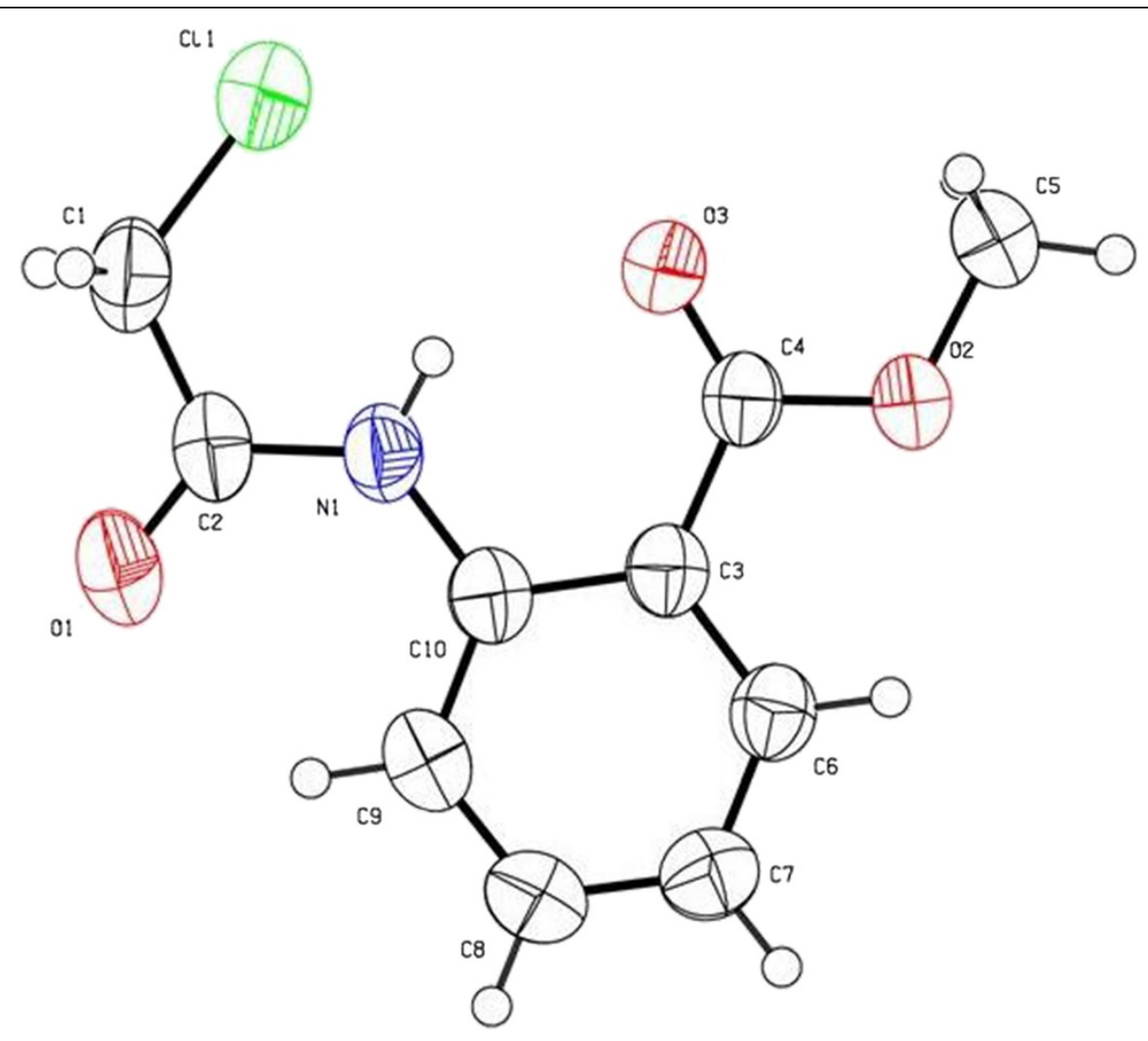

Figure 1 X-ray single crystal structure determined for compound 3 (CCDC 916675) [46].

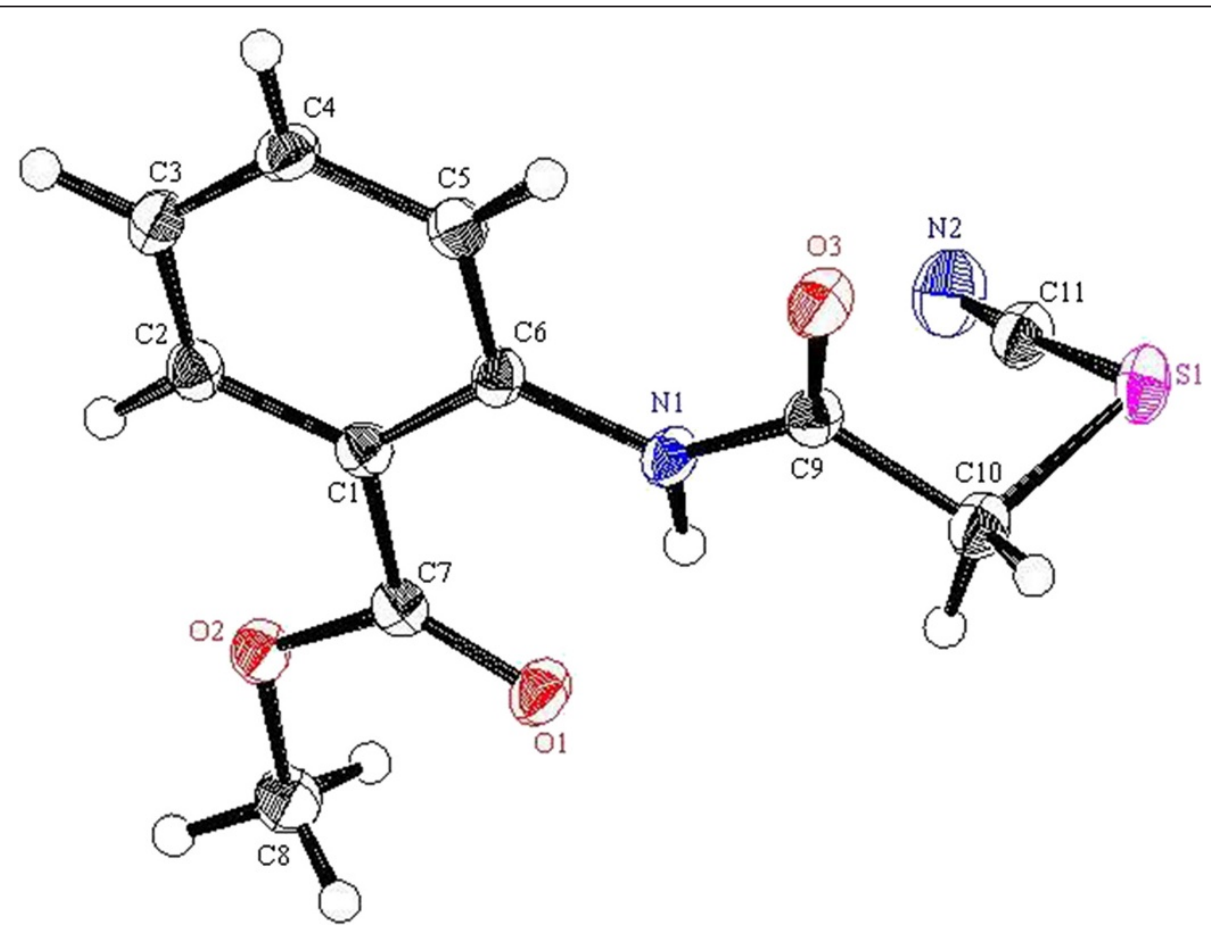

Figure 2 X-ray single crystal structure determined for compound 4 (CCDC 916665) [47]. 


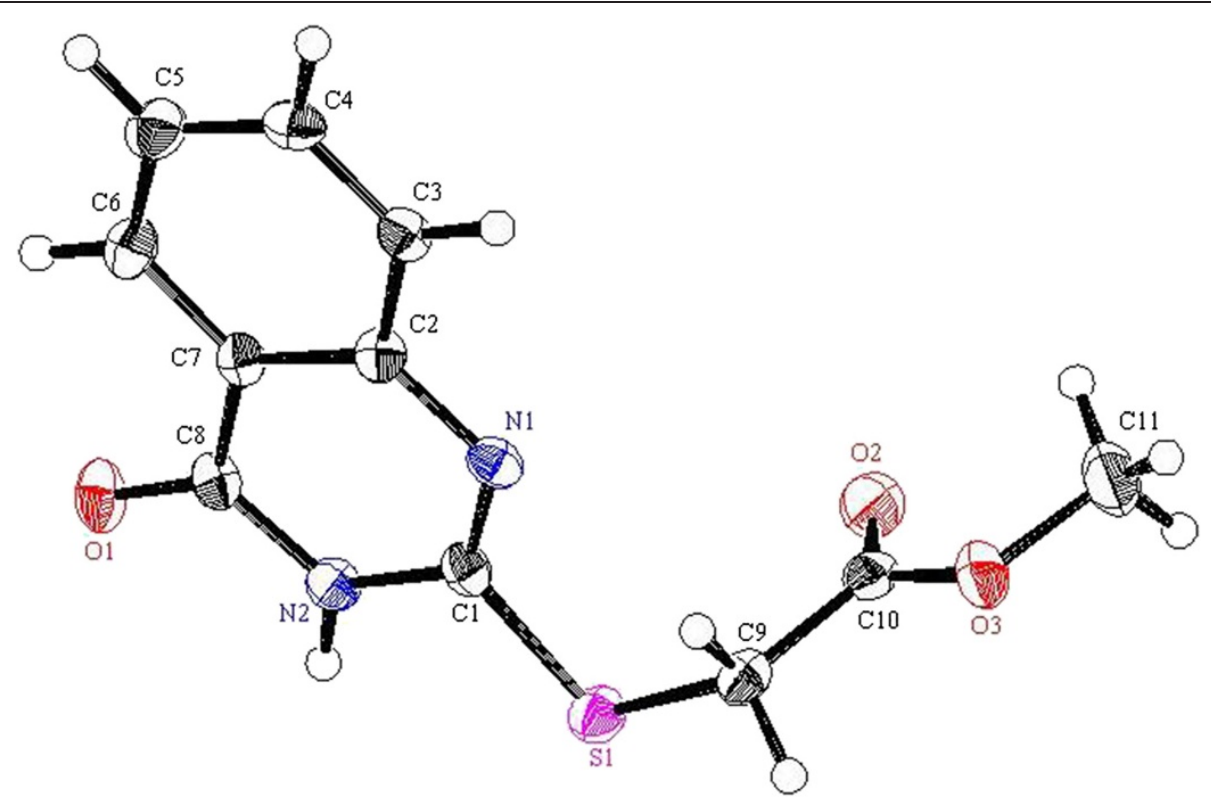

Figure 3 X-ray single crystal structure determined for compound 5 (CCDC 916666) [48].

the $\mathrm{DABCO}$ afford $9 \mathrm{a}$ in very good yields, whereas other bases, such as piperidine, morpholine, DBU, L-proline and $\mathrm{K}_{2} \mathrm{CO}_{3}$ were less effective. Then we probed the influence of different solvents on the reaction (entries 7-10). EtOH was found to be an effective solvent for good results. $\mathrm{CH}_{3} \mathrm{CN}$, DMF, dioxane and $\mathrm{MeOH}$ were found to be less effective. With the optimized reaction conditions in hand, we then explored the scope and generality of the synthesizing thiazolo[3,2-a]quinazoline derivatives 9 via Michael type addition reaction followed by the interamolecular cyclization. In addition we optimized the quantity of the catalyst added, as we found that $10 \mathrm{~mol} \%$ of DABCO is the best quantity for the reaction yield and we found that as the quantity of the added catalyst increased or decreased the yield of the reaction become lowered (Table 2).

In order to generate an alternative route for the synthesis of the above thiazolo[3,2-a]quinazoline 9 we conduct a reaction between methyl-2-(2-thiocyanatoacetamido) benzoate (4) and aromatic aldehydes under the same reaction conditions (EtOH, DABCO), but the structure of the obtained product was identified as ethyl-2-[(4-oxo-3,4dihydroquinazolin-2-yl)thio]acetate (10) [45] and not as 9, based on the spectrometric analyses and from the X-ray single crystal structure determination (cf. Scheme 4, Figure 9). Moreover refluxing $\mathbf{4}$ in ethanol only give $\mathbf{1 0}$ in $71 \%$ yields while refluxing it in ethanol containing DABCO affords 10 in $99 \%$ yields so the presence of the

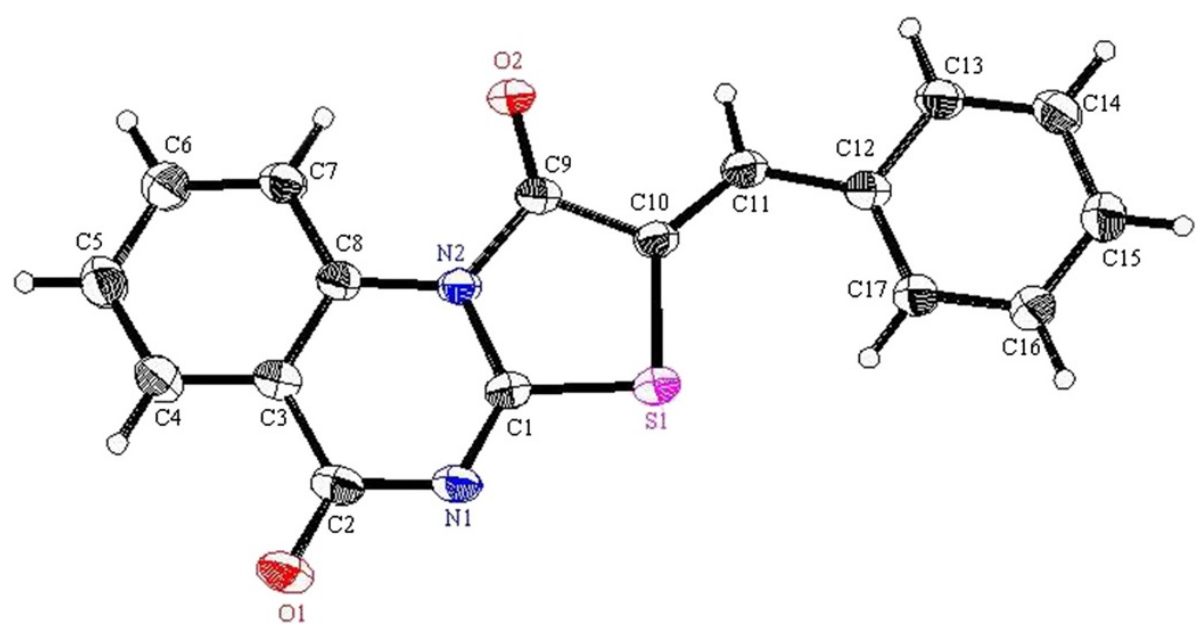

Figure 4 X-ray single crystal structure determined for compound 9a (CCDC 916667) [49]. 


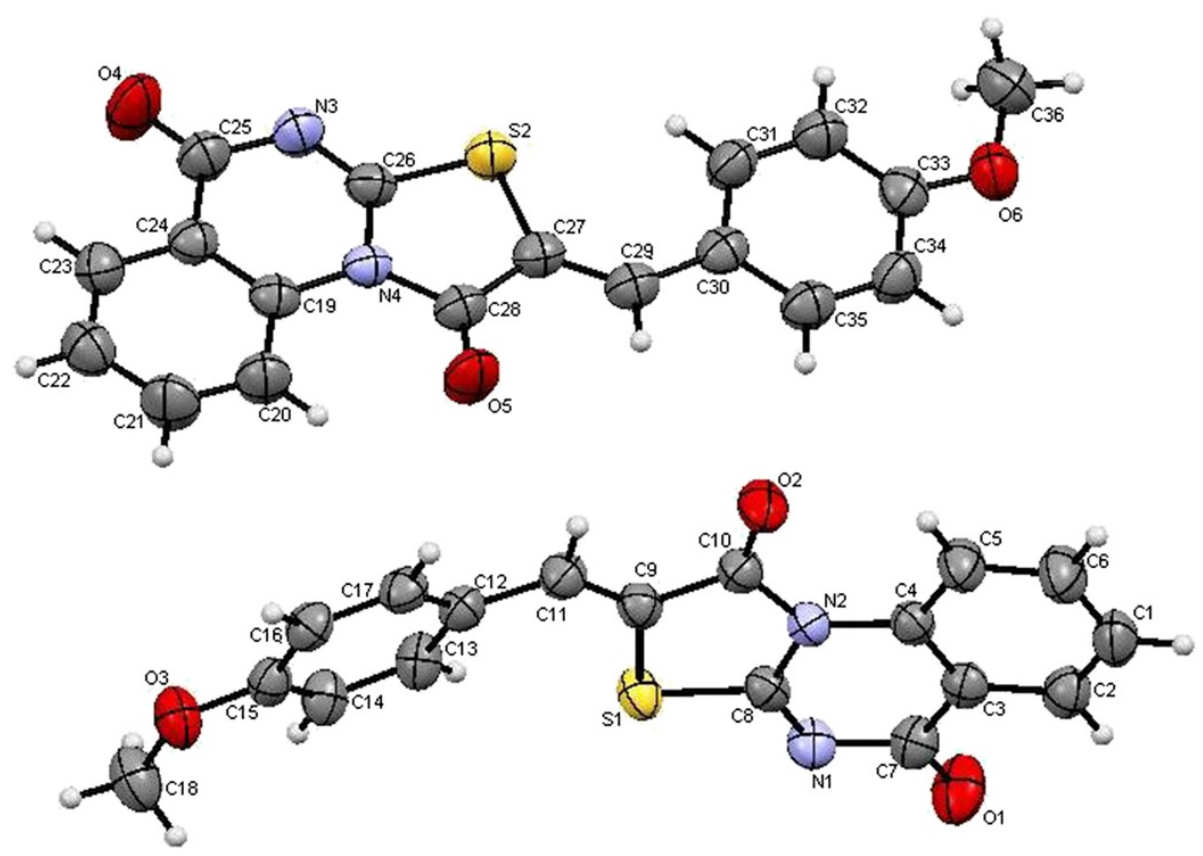

Figure 5 X-ray single crystal structure determined for compound 9c (CCDC 916674) [50].

DABCO enhance the reaction yield. Also the solvent has an effect on the reaction product since refluxing 4 in ethanol give $\mathbf{1 0}$ while refluxing $\mathbf{4}$ in methanol give $\mathbf{5}$ (cf. Scheme 2). Although the reaction between 10 and arylidene malononitriles does not take place conducting reaction between $\mathbf{1 0}$ and aromatic aldehydes in acetic acid containing sodium acetate afforded two regioselectively products with identical percentage yield as indicated from the ${ }^{1} \mathrm{H}$-NMR spectra, these two products were identified as $\mathbf{9}$ and $\mathbf{1 1}$ respectively, this mean that the regioselectively for this reaction is $50 \%$ for each route of cyclization.

The obtained ethyl-2-[(4-oxo-3,4-dihydroquinazolin-2-yl) thio]acetate (10) seem interesting precursor for the synthesis of a variety of a novel quinazoline and fused quinazoline derivatives. Thus reacting $\mathbf{1 0}$ with dimethylformamide dimethylacetal (DMF-DMA) yield also two regioselectively products with identical percentage yield, these two products were identified as $\mathbf{1 2}$ and $\mathbf{1 3}$ respectively, the structure of these two products was confirmed via the X-ray single crystal determination (cf. Scheme 5, Figures 10, 11).

Moreover reacting $\mathbf{1 0}$ with hydrazine hydrate in refluxing ethanol affording the corresponding hydrazide derivatives 14 which on reaction with aromatic aldehydes affording the corresponding condensation product 15, also 10 reacts with primary aromatic amines to

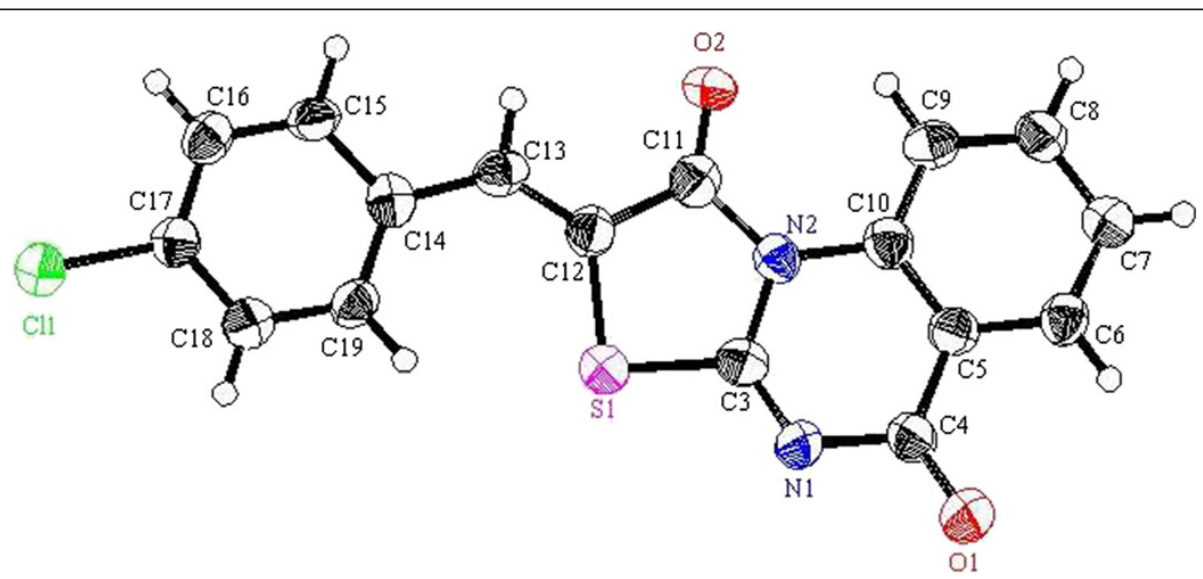

Figure 6 X-ray single crystal structure determined for compound 9d (CCDC 916671) [51]. 


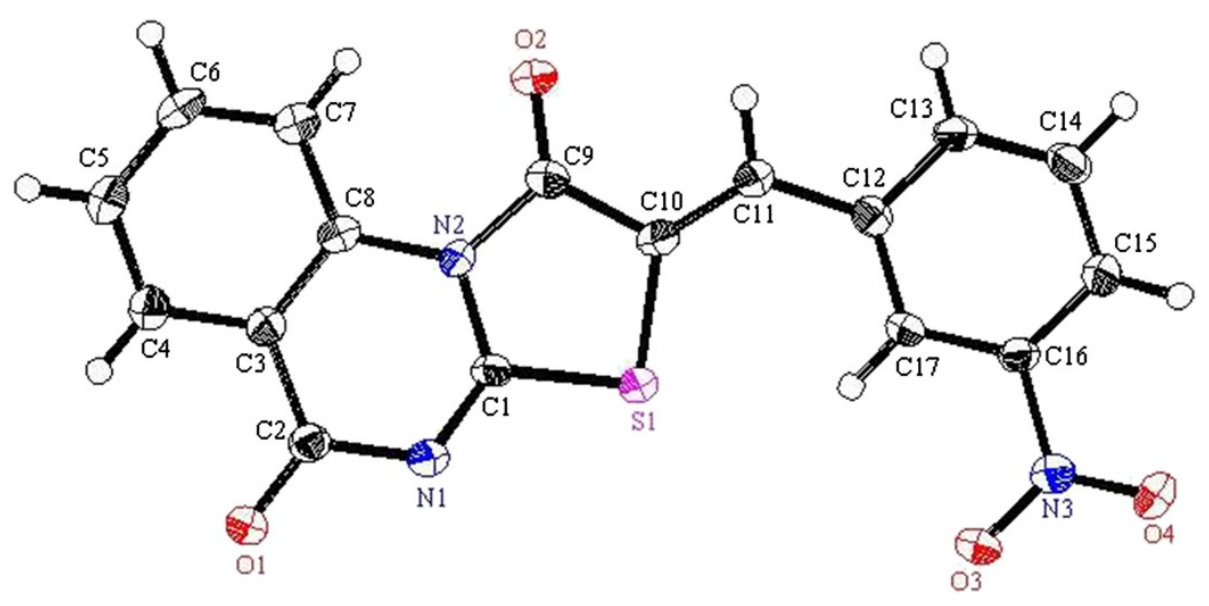

Figure 7 X-ray single crystal structure determined for compound 9f (CCDC 916670) [52].

afford the corresponding acetamide derivatives 16. In addition the triazolylquinazoline derivative 18 was formed via reaction of the hydrazide derivatives 14 with carbon disulfide in alcoholic potassium hydroxide to form 17 which on reaction with hydrazine hydrate affords the triazole 18. Moreover the quinazoline ethyl ester 10 was reacted with ethanol amine to afford the corresponding quinazoline hydroxyl derivative 19 (cf. Scheme 6).

\section{Experimental}

\section{General}

Melting points were recorded on a Griffin melting point apparatus and are reported uncorrected. IR spectra were recorded using $\mathrm{KBr}$ disks using a Perkin-Elmer System 2000 FT-IR spectrophotometer. ${ }^{1} \mathrm{H}-\mathrm{NMR}$ (400 MHz) or (600 MHz) and ${ }^{13} \mathrm{C}-\mathrm{NMR}(100 \mathrm{MHz})$ or $(150 \mathrm{MHz})$ spectra were recorded at $25^{\circ} \mathrm{C}$ in $\mathrm{CDCl}_{3}$ or DMSO- $d_{6}$ as solvent with TMS as internal standard on a Bruker DPX 400 or 600 super-conducting NMR spectrometer. Chemical shifts are reported in ppm. Mass spectra and HRMS were measured using a high resolution GC-MS (DFS) thermo spectrometers with EI (70 EV). Follow up of the reactions and checking homogeneity of the prepared compounds was made by thin layer chromatography (TLC). The crystal structures were determined by a Rigaku R-AXIS RAPID diffractometer and Bruker X8 Prospector and the data were collected at a temperature of $20 \pm 1^{\circ} \mathrm{C}$ to a maximum $2 \theta$ value of $55.0^{\circ}$ or $66.61^{\circ}$ using the $\omega$ scanning technique. The structure was solved either by direct method using SHELXS-97 (Sheldrick, 2008) and refined by Full-matrix leastsquares on $\mathrm{F}^{2}$. The non-hydrogen atoms were refined anisotropically. Data were corrected for absorption effects using the multi-scan method (SADABS) or by

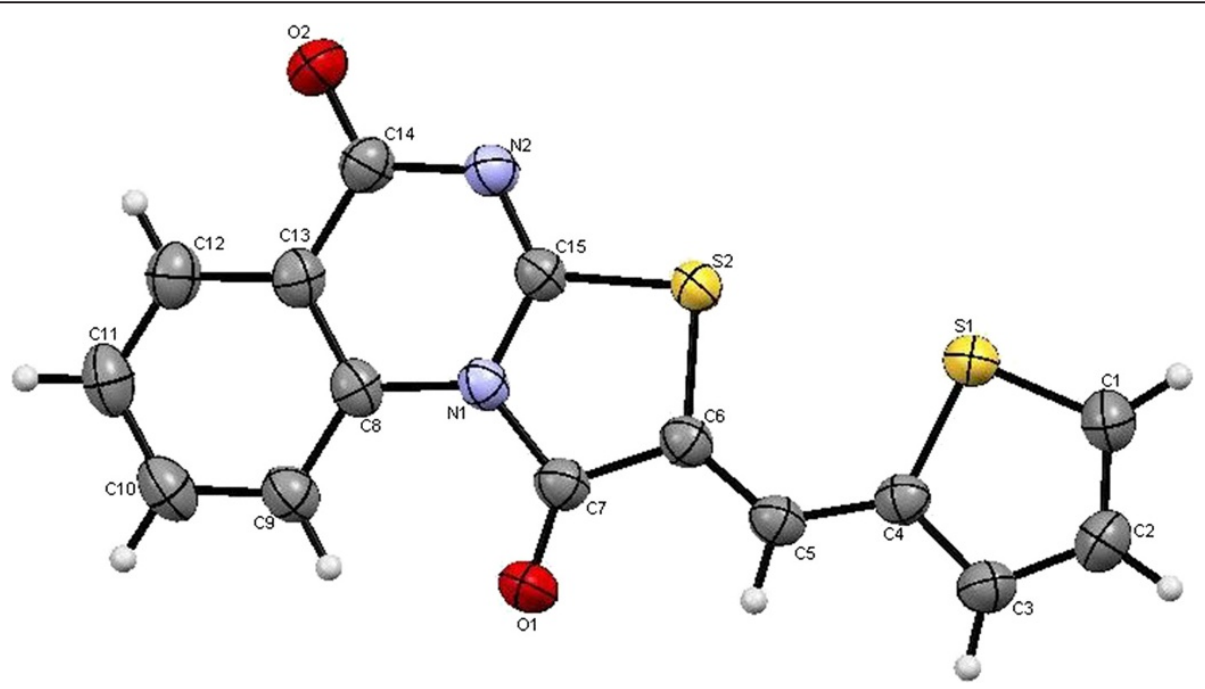

Figure 8 X-ray single crystal structure determined for compound 9h (CCDC 916673) [53]. 
<smiles>CC(=O)c1ccccc1NC(=O)CSC#N</smiles>

4<smiles>CC(=O)c1ccccc1NC(=O)C(CCBr)C(C)CBr</smiles>

4<smiles></smiles>

9<smiles>CCOC(=O)[Ba]C=C(C#N)C#N</smiles>

8a-i<smiles>CC[Se][As](CCCC(Br)=C(C#N)C#N)C(=O)Nc1ccccc1C(C)=O</smiles>

A<smiles>COC(=O)c1ccccc1N1C(=N)S/C(=C\Br)C1=O</smiles>

D<smiles>O=c1nc2s/c(=C\[Al])c(=O)n2c2ccccc12</smiles><smiles>CC(=O)c1ccccc1NC(=O)C1CCCC(CC(C#N)C#N)C1(Br)C[Si]C#N</smiles>

B

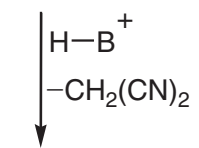<smiles>CCS/C(=C\Br)C(=O)Nc1ccccc1C(=O)OC</smiles>

C

8,

Scheme 3 Synthesis and the mechanistic pathway for the thiazolo[3,2-a]quinazoline derivatives 9.

charge flipping method and expanded using Fourier techniques. The non-hydrogen atoms were refined anisotropically. Hydrogen atoms were refined using the riding model

\section{Methyl-4-(2-chloroacetamido)benzoate (3)}

A solution of methyl anthranilate (1) $(10 \mathrm{mmol}, 1.52 \mathrm{~g})$ and chloroacetyl chloride (2) (1.36 g, $12 \mathrm{mmol})$ in chloroform $(50 \mathrm{~mL})$ was refluxed in the presence of $\mathrm{K}_{2} \mathrm{CO}_{3}$ (2.1 g, $\left.15 \mathrm{mmole}\right)$ for $3 \mathrm{~h}$. Then the solvent was removed in vасио and the residue was stirred with water
(100 mL) and filtered. The solid product is then washed with $5 \% \mathrm{NaHCO}_{3}$ solution and subsequently with water. The crude product was dried and recrystallized from $\mathrm{EtOH}$ as white crystals, yield: $96 \%$, m.p. $90-91^{\circ} \mathrm{C}$; IR (KBr): $v / \mathrm{cm}^{-1} 3225(\mathrm{NH}), 1699,1679$ (2CO); ${ }^{1} \mathrm{H}-\mathrm{NMR}$ $\left(\mathrm{DMSO}-d_{6}\right): \delta=3.89\left(\mathrm{~s}, 3 \mathrm{H}, \mathrm{CH}_{3}\right), 4.45\left(\mathrm{~s}, 2 \mathrm{H}, \mathrm{CH}_{2}\right)$, $7.25(\mathrm{t}, J=8.0 \mathrm{~Hz}, 1 \mathrm{H}, \mathrm{Ar}-\mathrm{H}), 7.66(\mathrm{t}, J=8.0 \mathrm{~Hz}, 1 \mathrm{H}$, Ar-H), 7.98 (d, $J=8.0 \mathrm{~Hz}, 1 \mathrm{H}, \mathrm{Ar}-\mathrm{H}), 8.40$ (d, $J=8.0$ $\mathrm{Hz}, 1 \mathrm{H}, \mathrm{Ar}-\mathrm{H})$ and $11.34 \mathrm{ppm}(\mathrm{s}, 1 \mathrm{H}, \mathrm{NH}) ;{ }^{13} \mathrm{C}-\mathrm{NMR}$ $\left(\mathrm{DMSO}-d_{6}\right): \delta=43.3\left(\mathrm{CH}_{2}\right), 52.5\left(\mathrm{CH}_{3}\right), 116.8,120.4$, 123.7, 130.7, 134.2, 139.2, 165.1 and 167.4 ppm (Ar-C 
Table 1 Optimization of conditions of the synthesis $9 a^{a}$

\begin{tabular}{ccccc}
\hline Entry $^{\text {a }}$ & Base & Solvent & Time (min) & Yield (\%) \\
\hline 1 & DABCO & EtOH & 5 & 81 \\
\hline 2 & piperidine & EtOH & 30 & 67 \\
\hline 3 & morpholine & $\mathrm{EtOH}$ & 25 & 61 \\
\hline 4 & $\mathrm{DBU}$ & $\mathrm{EtOH}$ & 15 & 55 \\
\hline 5 & $\mathrm{~L}-$ proline & $\mathrm{EtOH}$ & 40 & 69 \\
\hline 6 & $\mathrm{~K}_{2} \mathrm{CO}_{3}$ & $\mathrm{EtOH}$ & 60 & none \\
\hline 7 & $\mathrm{DABCO}$ & $\mathrm{CH}_{3} \mathrm{CN}$ & 15 & 35 \\
\hline 8 & $\mathrm{DABCO}$ & $\mathrm{DMF}$ & 5 & 46 \\
\hline 9 & $\mathrm{DABCO}$ & Dioxane & 5 & 51 \\
\hline 10 & $\mathrm{DABCO}$ & $\mathrm{MeOH}$ & 5 & 68 \\
\hline
\end{tabular}

${ }^{a}$ Reaction conditions: methyl-2-(2-thiocyanatoacetamido)benzoate (4) (5 $\mathrm{mmol})$, benzylidene malononitriles $8 \mathbf{a}(5 \mathrm{mmol})$, and base $(10 \mathrm{~mol} \%)$ in solvent $(25 \mathrm{~mL})$ under reflux for the given time.

and CO); $\mathrm{MS}$ (EI): $m / z(\%) 229\left(\mathrm{M}^{+}+2,14.12\right), 228$ $\left(\mathrm{M}^{+}+1,7.85\right), 227\left(\mathrm{M}^{+}, 43.55\right)$; HRMS (EI): $m / z$ calcd. for $\mathrm{C}_{10} \mathrm{H}_{10}^{35} \mathrm{ClNO}_{3}\left(\mathrm{M}^{+}\right)$227.0343, found 227.0343. Crystal data: $\mathrm{C}_{10} \mathrm{H}_{10} \mathrm{ClNO}_{3}, \mathrm{M}=227.65$, monoclinic, $\mathrm{a}=12.8980(12) \AA, \mathrm{b}=4.6089(4) \AA, \mathrm{c}=18.2514(15) \AA, \mathrm{V}$ $=1031.08(16) \AA^{3}, \alpha=\gamma=90.00^{\circ}, \beta=108.133(5)$, space group: P $121 / \mathrm{c} 1, Z=4, D_{\text {calc }}=1.466 \mathrm{Mg} \mathrm{cm}^{-3}$, No. of reflections measured $4589,2 \theta_{\max }=66.59^{\circ}, \mathrm{R} 1=0.034$. Figure 1 illustrates the structure as determined. Full data can be obtained on request from the CCDC [46].

\section{Methyl-2-(2-thiocyanatoacetamido)benzoate (4)}

A solution of methyl-4-(2-chloroacetamido)benzoate (3) $(2.27 \mathrm{~g}, 10 \mathrm{mmol})$ and ammonium thiocyanate (15 $\mathrm{mmol})$ in acetone or absolute methanol $(30 \mathrm{~mL})$ was refluxed for $6 \mathrm{~h}$ and allowed to cool. The formed precipitate was filtered off, washed with water and then recrystallised from $\mathrm{MeOH}$ as white crystals, yield: 92\%, m.p. $109-110^{\circ} \mathrm{C}$; IR (KBr): $v / \mathrm{cm}^{-1} 3241(\mathrm{NH}), 2155$ (SCN), 1737, 1698 (2CO); ${ }^{1} \mathrm{H}-\mathrm{NMR}$ (DMSO- $\left.d_{6}\right): \delta=$ $3.87\left(\mathrm{~s}, 3 \mathrm{H}, \mathrm{CH}_{3}\right), 4.24\left(\mathrm{~s}, 2 \mathrm{H}, \mathrm{CH}_{2}\right), 7.25(\mathrm{t}, J=8.0 \mathrm{~Hz}$, $1 \mathrm{H}, \mathrm{Ar}-\mathrm{H}), 7.63$ (t, $J=8.0 \mathrm{~Hz}, 1 \mathrm{H}, \mathrm{Ar}-\mathrm{H}), 7.91$ (d, $J=8.0$ $\mathrm{Hz}, 1 \mathrm{H}, \mathrm{Ar}-\mathrm{H}), 8.16(\mathrm{~d}, J=8.0 \mathrm{~Hz}, 1 \mathrm{H}, \mathrm{Ar}-\mathrm{H})$ and 10.93 ppm $(\mathrm{s}, 1 \mathrm{H}, \mathrm{NH}) ;{ }^{13} \mathrm{C}-\mathrm{NMR}\left(\mathrm{DMSO}-d_{6}\right): \delta=37.4\left(\mathrm{CH}_{2}\right)$,

Table 2 Optimization mol \% of DABCO during the synthesis 9a

\begin{tabular}{ccc}
\hline Entry $^{\text {a }}$ & mol \% of DABCO & Yield (\%) \\
\hline 1 & 5 & 75 \\
\hline 2 & 10 & 81 \\
\hline 3 & 15 & 73 \\
\hline 4 & 20 & 70 \\
\hline 5 & 30 & 62 \\
\hline 6 & 40 & 54 \\
\hline 7 & 50 & 46 \\
\hline 8 & 60 & 45 \\
\hline 9 & 70 & 45 \\
\hline
\end{tabular}

$52.5\left(\mathrm{CH}_{3}\right), 112.7(\mathrm{SCN}), 118.8,121.7,124.1,130.6$, 133.9, 138.4, 164.6 and $167.2 \mathrm{ppm}$ (Ar-C and CO); MS (EI): $m / z(\%) 251\left(\mathrm{M}^{+}+1,10.42\right), 250\left(\mathrm{M}^{+}, 34.91\right)$; HRMS (EI): $m / z$ calcd. for $\mathrm{C}_{11} \mathrm{H}_{10} \mathrm{~N}_{2} \mathrm{O}_{3} \mathrm{~S}\left(\mathrm{M}^{+}\right)$250.0406, found 250.0407. Crystal data: $\mathrm{C}_{11} \mathrm{H}_{10} \mathrm{~N}_{2} \mathrm{O}_{3} \mathrm{~S}, \mathrm{M}=250.28$, triclinic, $\mathrm{a}=6.7349(6) \AA, \mathrm{b}=8.3058(9) \AA, \mathrm{c}=10.3398(8)$ $\AA, V=558.19(9) \AA^{3}, \alpha=86.526(6), \beta=85.256(6)^{\circ}, \gamma=$ 75.747 (6), space group: $\mathrm{P}-1$ (\#2), $\mathrm{Z}=2, \mathrm{D}_{\text {calc }}=1.489 \mathrm{Mg}$

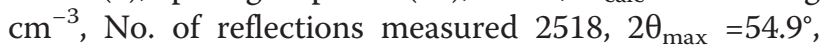
$\mathrm{R} 1=0.033$. Figure 2 illustrates the structure as determined. Full data can be obtained on request from the CCDC [47].

Methyl-2-(4-oxo-3,4-dihydroquinazolin-2-ylthio)acetate (5) A solution of methyl-4-(2-chloroacetamido)benzoate (3) (2.27 g, $10 \mathrm{mmol})$ and ammonium thiocyanate $(15 \mathrm{mmol})$ in methanol $(30 \mathrm{~mL})$ was refluxed for $12 \mathrm{~h}$, or refluxing 4 in $\mathrm{MeOH}$ for $6 \mathrm{~h}$ then the reaction mixture was allowed to cool down to room temperature. The formed precipitate was filtered off, washed with water and then recrystallised from $\mathrm{MeOH}$ as white crystals, yield: $88 \%$, m.p. $191-192^{\circ} \mathrm{C}$; IR (KBr): $v / \mathrm{cm}^{-1} 3172(\mathrm{NH}), 1737,1686(2 \mathrm{CO}) ;{ }^{1} \mathrm{H}-\mathrm{NMR}$ $\left(\mathrm{DMSO}-d_{6}\right): \delta=3.69\left(\mathrm{~s}, 3 \mathrm{H}, \mathrm{CH}_{3}\right), 4.11\left(\mathrm{~s}, 2 \mathrm{H}, \mathrm{CH}_{2}\right)$, 7.41-7.45 (m, 2H, Ar-H), $7.76(\mathrm{t}, J=7.6 \mathrm{~Hz}, 1 \mathrm{H}, \mathrm{Ar}-\mathrm{H})$, $8.03(\mathrm{~d}, J=7.6 \mathrm{~Hz}, 1 \mathrm{H}, \mathrm{Ar}-\mathrm{H}), 12.72 \mathrm{ppm}(\mathrm{s}, 1 \mathrm{H}, \mathrm{NH})$; ${ }^{13} \mathrm{C}-\mathrm{NMR}\left(\mathrm{DMSO}-d_{6}\right): \delta=32.1\left(\mathrm{CH}_{2}\right), 52.4\left(\mathrm{CH}_{3}\right), 119.8$, $125.8,125.9,126.0,134.7,148.1,154.6,161.1$ and 169.0 ppm (Ar-C and CO); MS (EI): $m / z$ (\%) $251\left(\mathrm{M}^{+}+1,7.24\right)$, $250\left(\mathrm{M}^{+}, 32.55\right)$; HRMS (EI): $m / z$ calcd. for $\mathrm{C}_{11} \mathrm{H}_{10} \mathrm{~N}_{2} \mathrm{O}_{3} \mathrm{~S}$ $\left(\mathrm{M}^{+}\right)$250.0406, found 250.0406. Crystal data: $\mathrm{C}_{11} \mathrm{H}_{10} \mathrm{~N}_{2} \mathrm{O}_{3} \mathrm{~S}, \mathrm{M}=250.28$, orthorhombic, $\mathrm{a}=17.863(2)$ $\AA, \mathrm{b}=13.1670(7) \AA, \mathrm{c}=4.6272(2) \AA, \mathrm{V}=1088.3(1) \AA^{3}$, $\alpha=\beta=\gamma=90.0^{\circ}$, space group: Pna21 (\#33), $Z=4, D_{\text {calc }}=$ $1.527 \mathrm{Mg} \mathrm{cm}^{-3}$, No. of reflections measured 2058, $2 \theta_{\max }=$ $54.80^{\circ}, \mathrm{R} 1=0.0384$. Figure 3 illustrates the structure as determined. Full data can be obtained on request from the CCDC [48].

\section{General procedure for the synthesis of thiazolo[3,2-a]- quinazoline derivatives $9 a-i$}

Independent mixtures of methyl-2-(2-thiocyanatoacetamido)benzoate (4) (1.25 g, $5 \mathrm{mmol}$ ) and the appropriate arylidene malononitrile 8a-i $(5 \mathrm{mmol})$ in ethanol $(25 \mathrm{~mL})$ containing DABCO $(0.11 \mathrm{~g}, 10 \mathrm{~mol} \%)$ were stirred at reflux for $5 \mathrm{~min}$. Then, the reaction mixtures were allowed to cool down to room temperature. The solid which formed was collected by filtration, washed with hot ethanol, and recrystallized from the appropriate solvent to afford $9 \mathbf{a}-\mathbf{i}$ respectively, as pure substances.

\section{(Z)-2-Benzylidene-2H-thiazolo[3,2-a]quinazoline-1,5-dione (9a)}

Recrystallized from an EtOH/ dioxane (1:1) mixture as canary yellow crystals, yield: (81\%), m.p. $236-237^{\circ} \mathrm{C}$; IR (KBr): $v / \mathrm{cm}^{-1} 1717,1672$ (2CO); ${ }^{1} \mathrm{H}-\mathrm{NMR}$ (DMSO- $d_{6}$ ): 


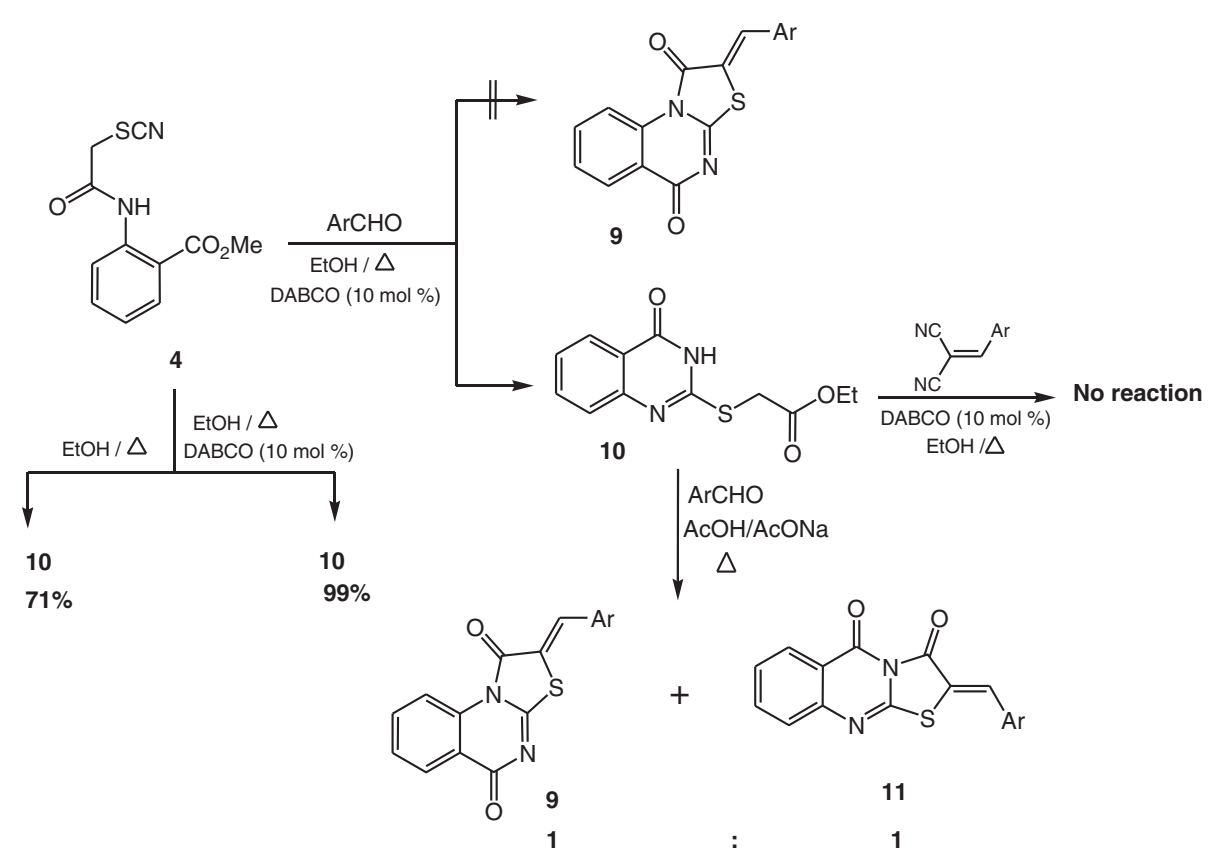

Scheme 4 Reaction of 10 with aromatic aldehydes.

$\delta=7.54-7.68(\mathrm{~m}, 4 \mathrm{H}, \mathrm{Ar}-\mathrm{H}), 7.76\left(\mathrm{~d}, J=7.6 \mathrm{~Hz}, 2 \mathrm{H}, \quad\right.$ and CO); MS (EI): $m / z(\%) 307\left(\mathrm{M}^{+}+1,19.44\right), 306\left(\mathrm{M}^{+}\right.$, Ar-H), $7.94(\mathrm{t}, J=8.0 \mathrm{~Hz}, 1 \mathrm{H}, \mathrm{Ar}-\mathrm{H}), 8.15(\mathrm{~d}, J=8.0 \mathrm{~Hz}, \quad 100)$; HRMS (EI): $\mathrm{m} / z$ calcd. for $\mathrm{C}_{17} \mathrm{H}_{10} \mathrm{~N}_{2} \mathrm{O}_{2} \mathrm{~S}\left(\mathrm{M}^{+}\right)$ $1 \mathrm{H}, \mathrm{Ar}-\mathrm{H}), 8.17(\mathrm{~s}, 1 \mathrm{H}$, olefinic $\mathrm{CH})$ and $8.98 \mathrm{ppm}\left(\mathrm{d}, J\right.$ 306.0457, found 306.0457. Crystal data: $\mathrm{C}_{17} \mathrm{H}_{10} \mathrm{~N}_{2} \mathrm{O}_{2} \mathrm{~S}$, $=8.0 \mathrm{~Hz}, 1 \mathrm{H}, \mathrm{Ar}-\mathrm{H}) ;{ }^{13} \mathrm{C}-\mathrm{NMR}\left(\mathrm{DMSO}-d_{6}\right): \delta=116.3, \quad \mathrm{M}=306.34$, triclinic, $\mathrm{a}=6.391(5) \AA, \mathrm{b}=8.650(7) \AA, \mathrm{c}=$ $117.7,118.8,127.9,127.9,129.6,130.5,131.3,132.7, \quad 12.77(1) \AA, V=676.2(9) \AA^{3}, \alpha=76.57(2), \beta=87.89(1), \gamma=$ 134.5, 135.8, 137.1, 164.2, 164.3 and $165.2 \mathrm{ppm}$ (Ar-C $\quad 79.99(1)^{\circ}$, space group: $\mathrm{P}-1$ (\#2), $\mathrm{Z}=2$, $\mathrm{D}_{\text {calc }}=1.504 \mathrm{Mg}$

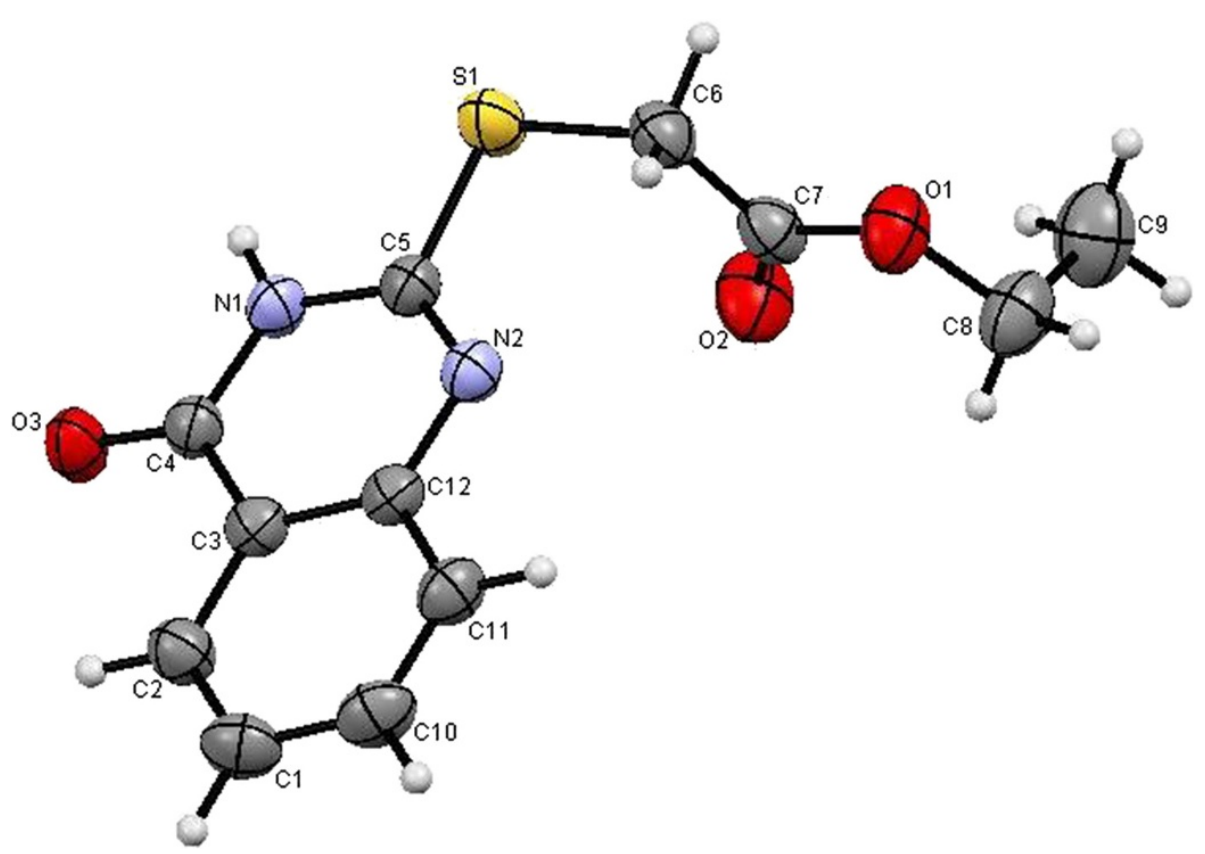

Figure 9 X-ray single crystal structure determined for compound 10 (CCDC 916672) [54]. 
<smiles>CCOC(=O)CSc1nc2ccccc2c(=O)[nH]1</smiles><smiles>CCOC(=O)/C(=C\N(C)C)Sc1nc2ccccc2c(=O)[nH]1</smiles><smiles>CN(C)/C=c1\sc2nc(=O)c3ccccc3n2c1=O</smiles>

$43 \%$<smiles>CCOC(=O)c1cn2c(=O)c3ccccc3nc2s1</smiles>

$43 \%$

Scheme 5 Reaction of 10 with dimethylformamide dimethylacetal (DMF-DMA).

$\mathrm{cm}^{-3}$, No. of reflections measured 5937, $2 \theta_{\max }=52.70^{\circ}$, $\mathrm{R} 1=0.0458$. Figure 4 illustrates the structure as determined. Full data can be obtained on request from the CCDC [49].

\section{(Z)-2-(4-Methylbenzylidene)-2H-thiazolo[3,2-a]quinazoline- \\ 1,5-dione (9b)}

Recrystallized from dioxane as pale yellow crystals, yield: (79\%), m.p. $253-254^{\circ} \mathrm{C}$; IR (KBr): $v / \mathrm{cm}^{-1} 1726,1681$ (2CO); ${ }^{1} \mathrm{H}-\mathrm{NMR}\left(\mathrm{DMSO}-d_{6}\right): \delta=2.40\left(\mathrm{~s}, 3 \mathrm{H}, \mathrm{CH}_{3}\right), 7.40$ (d, $J=7.6 \mathrm{~Hz}, 2 \mathrm{H}, \mathrm{Ar}-\mathrm{H}), 7.59-7.64(\mathrm{~m}, 3 \mathrm{H}, \mathrm{Ar}-\mathrm{H}), 7.90$ $(\mathrm{t}, J=8.0 \mathrm{~Hz}, 1 \mathrm{H}, \mathrm{Ar}-\mathrm{H}), 8.10(\mathrm{~s}, 1 \mathrm{H}$, olefinic $C H), 8.16$ $(\mathrm{d}, J=8.0 \mathrm{~Hz}, 1 \mathrm{H}, \mathrm{Ar}-\mathrm{H})$ and $8.97 \mathrm{ppm}(\mathrm{d}, J=8.0 \mathrm{~Hz}$, $1 \mathrm{H}, \mathrm{Ar}-\mathrm{H}) ;{ }^{13} \mathrm{C}-\mathrm{NMR}$ (DMSO- $\left.d_{6}\right): \delta=21.2\left(\mathrm{CH}_{3}\right), 116.3$, $117.5,117.8,127.8,127.9,130.1,130.2,130.6,134.5$, 135.9, 137.1, 141.8, 164.2, 164.4 and 165.3 ppm (Ar-C and CO); $\mathrm{MS}$ (EI): $m / z(\%) 321\left(\mathrm{M}^{+}+1,17.99\right), 320$
$\left(\mathrm{M}^{+}, 100\right)$; HRMS (EI): $m / z$ calcd. for $\mathrm{C}_{18} \mathrm{H}_{12} \mathrm{~N}_{2} \mathrm{O}_{2} \mathrm{~S}$ $\left(\mathrm{M}^{+}\right)$320.0613, found 320.0614.

(Z)-2-(4-Methoxybenzylidene)-2H-thiazolo[3,2-a]quinazoline1,5-dione (9c)

Recrystallized from dioxane as yellow crystals, yield: (83\%), m.p. 261-262 ${ }^{\circ} \mathrm{C}$; IR (KBr): $v / \mathrm{cm}^{-1} 1721,1676$ (2CO); ${ }^{1} \mathrm{H}-$ NMR (DMSO- $\left.d_{6}\right): \delta=3.87\left(\mathrm{~s}, 2 \mathrm{H}, \mathrm{OCH}_{3}\right), 7.18(\mathrm{~d}, J=7.6$ $\mathrm{Hz}, 2 \mathrm{H}, \mathrm{Ar}-\mathrm{H}), 7.66$ (t, $J=8.0 \mathrm{~Hz}, 1 \mathrm{H}, \mathrm{Ar}-\mathrm{H}), 7.75$ (d, $J=$ $7.6 \mathrm{~Hz}, 2 \mathrm{H}, \mathrm{Ar}-\mathrm{H}), 7.93(\mathrm{t}, J=8.0 \mathrm{~Hz}, 1 \mathrm{H}, \mathrm{Ar}-\mathrm{H}), 8.11$ (s, $1 \mathrm{H}$, olefinic $C H), 8.17(\mathrm{~d}, J=8.0 \mathrm{~Hz}, 1 \mathrm{H}, \mathrm{Ar}-\mathrm{H})$ and 9.02 ppm $(\mathrm{d}, J=8.0 \mathrm{~Hz}, 1 \mathrm{H}, \mathrm{Ar}-\mathrm{H}) ;{ }^{13} \mathrm{C}-\mathrm{NMR}$ (DMSO- $d_{6}$ at $\left.80^{\circ} \mathrm{C}\right): \delta=56.2\left(\mathrm{OCH}_{3}\right), 115.8,116.8,118.6,126.0,128.2$, $128.4,130.9,133.1,134.6,136.8,137.6,162.5,164.2,164.6$ and $165.4 \mathrm{ppm}$ (Ar-C and CO); MS (EI): $m / z(\%) 337$ $\left(\mathrm{M}^{+}+1,16.96\right), 336\left(\mathrm{M}^{+}, 100\right)$; HRMS (EI): $m / z$ calcd. for $\mathrm{C}_{18} \mathrm{H}_{12} \mathrm{~N}_{2} \mathrm{O}_{3} \mathrm{~S}\left(\mathrm{M}^{+}\right)$336.0563, found 336.0563. Crystal data:

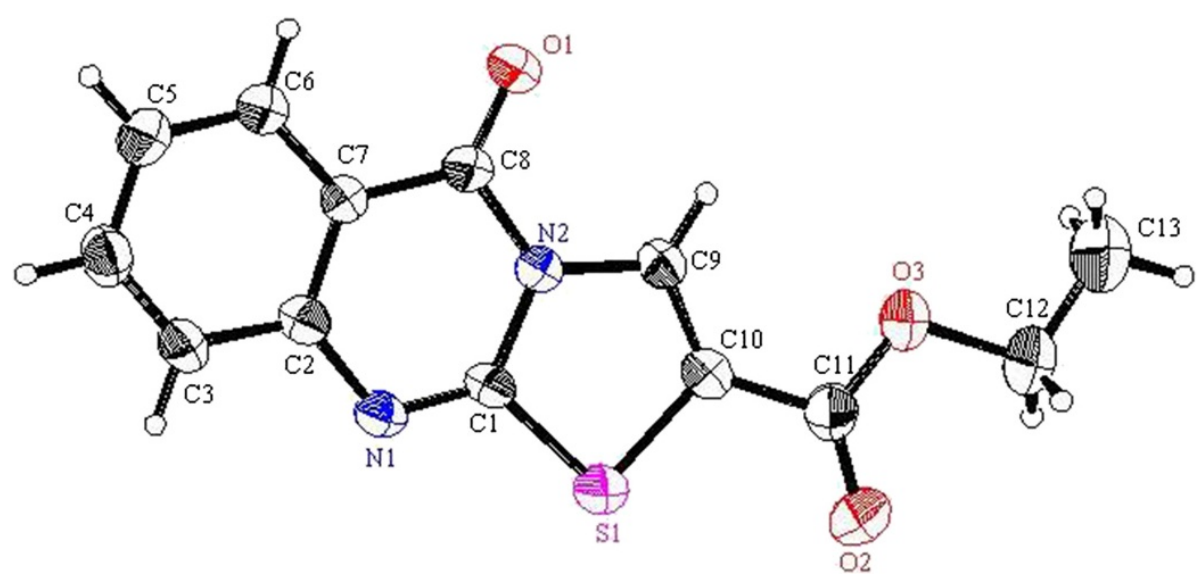

Figure 10 X-ray single crystal structure determined for compound 12 (CCDC 916668) [55]. 


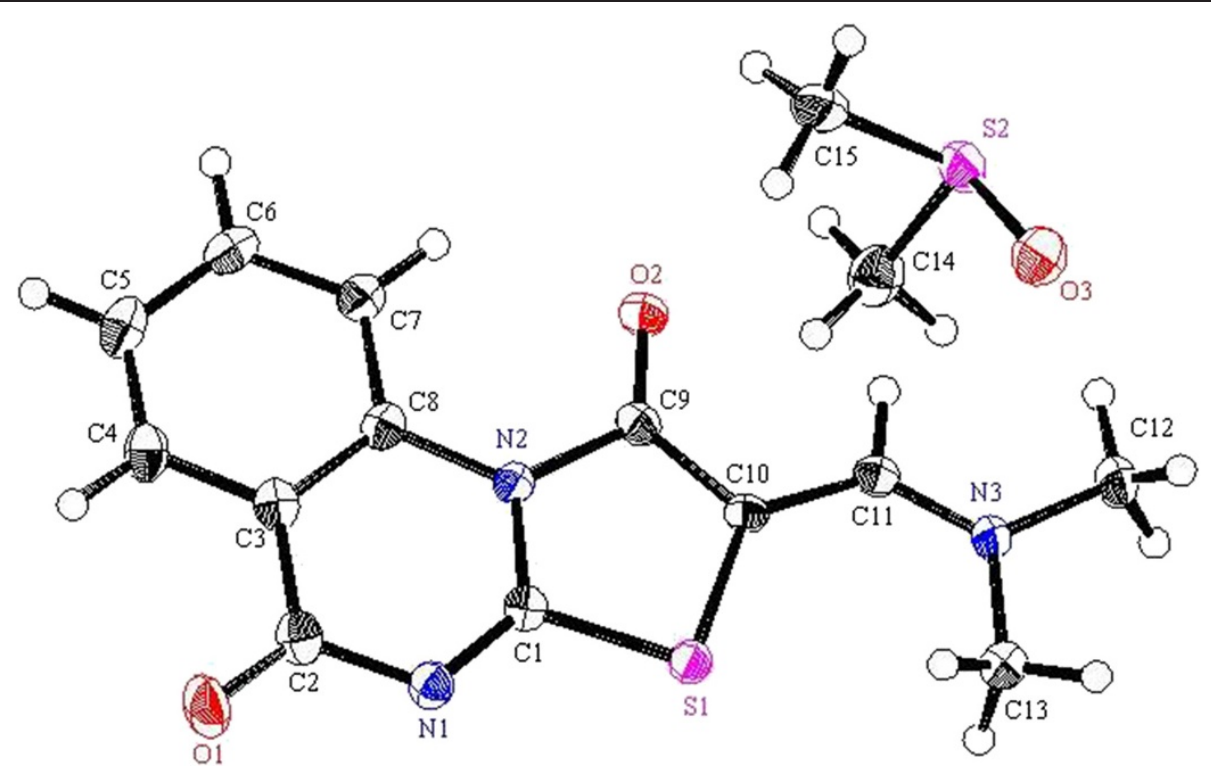

Figure 11 X-ray single crystal structure determined for compound 13 (CCDC 916669) [56].

$\mathrm{C}_{18} \mathrm{H}_{12} \mathrm{~N}_{2} \mathrm{O}_{3} \mathrm{~S}, \mathrm{M}=336.37$, monoclinic, $\mathrm{a}=8.1837(3) \AA$, $\mathrm{b}=15.2696(4) \AA, \mathrm{c}=24.0028(7) \AA, \mathrm{V}=2996.81(16) \AA^{3}$, $\alpha=\gamma=90.00^{\circ}, \beta=92.397(2)^{\circ}$, space group: $\mathrm{P} 121 / \mathrm{c} 1, \mathrm{Z}=$ $4, \mathrm{D}_{\text {calc }}=1.491 \mathrm{Mg} \mathrm{cm}^{-3}$, No. of reflections measured 5296, $2 \theta_{\max }=66.73^{\circ}, \mathrm{R} 1=0.0339$. Figure 5 illustrates the structure as determined. Full data can be obtained on request from the $\mathrm{CCDC}[50]$.
(Z)-2-(4-Chlorobenzylidene)-2H-thiazolo[3,2-a]quinazoline1,5-dione (9d)

Recrystallized from dioxane as yellow crystals, yield: (85\%), m.p. $279-280^{\circ} \mathrm{C}$; IR (KBr): $v / \mathrm{cm}^{-1} 1720,1692$ $(2 \mathrm{CO}) ;{ }^{1} \mathrm{H}-\mathrm{NMR}\left(\mathrm{DMSO}-d_{6}\right): \delta=7.63-7.66(\mathrm{~m}, 3 \mathrm{H}$, Ar-H), $7.75(\mathrm{~d}, J=8.4 \mathrm{~Hz}, 2 \mathrm{H}, \mathrm{Ar}-\mathrm{H}), 7.92(\mathrm{t}, J=8.4 \mathrm{~Hz}$, $1 \mathrm{H}, \mathrm{Ar}-\mathrm{H}), 8.14(\mathrm{~s}, 1 \mathrm{H}$, olefinic $C H), 8.20(\mathrm{~d}, J=8.4 \mathrm{~Hz}$,

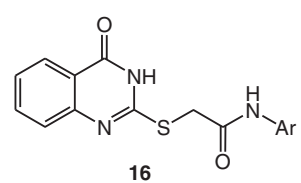

16<smiles>[14CH3]N</smiles>
$\stackrel{\mathrm{N}_{2} \mathrm{H}_{4} \cdot \mathrm{H}_{2} \mathrm{O}}{\longrightarrow}$<smiles>CCOC(=O)CSc1nc2ccccc2c(=O)[nH]1</smiles>
10<smiles>O=C(CSc1nc2cc(CCO)ccc2c(=O)[nH]1)NCCO</smiles>

19

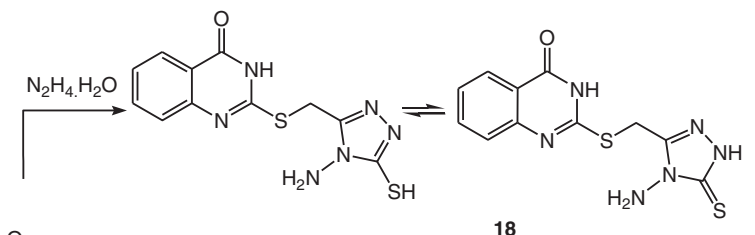<smiles>O=C(CSc1nc2ccccc2c(=O)[nH]1)NNC(=S)[Te]</smiles><smiles>[CH]1[CH]C=C1</smiles><smiles>O=C(CSc1nc2ccccc2c(=O)[nH]1)N/N=C/I</smiles> 
$1 \mathrm{H}, \mathrm{Ar}-\mathrm{H})$ and $8.97 \mathrm{ppm}(\mathrm{d}, J=8.4 \mathrm{~Hz}, 1 \mathrm{H}, \mathrm{Ar}-\mathrm{H}) ;{ }^{13} \mathrm{C}-$ NMR (DMSO- $d_{6}$ at $\left.80^{\circ} \mathrm{C}\right): \delta=116.3,117.7,119.6$, $127.9,129.5,129.6,131.6,132.10,134.4,134.5,135.8$, 137.0, 164.0, 164.2 and $165.2 \mathrm{ppm}$ (Ar-C and CO); MS (EI): $m / z(\%) 341\left(\mathrm{M}^{+}+1,20.28\right), 340\left(\mathrm{M}^{+}, 100\right)$; HRMS (EI): $m / z$ calcd. for $\mathrm{C}_{17} \mathrm{H}_{9}^{35} \mathrm{ClN}_{2} \mathrm{O}_{2} \mathrm{~S}\left(\mathrm{M}^{+}\right)$340.0067, found 340.0066. Crystal data: $\mathrm{C}_{17} \mathrm{H}_{9} \mathrm{ClN}_{2} \mathrm{O}_{2} \mathrm{~S}, \mathrm{M}=$ 340.78, monoclinic, $\mathrm{a}=3.8563(7) \AA, \mathrm{b}=12.784(2) \AA$, $c=28.101(5) \AA, V=1384.9(4) \AA^{3}, \alpha=\gamma=90.00^{\circ}, \beta=$ 91.456(7) ${ }^{\circ}$, space group: $\mathrm{P} 21 / \mathrm{c}(\# 14), \mathrm{Z}=4, \mathrm{D}_{\text {calc }}=1.634$ $\mathrm{Mg} \mathrm{cm}{ }^{-3}$, No. of reflections measured 7510, $2 \theta_{\max }=$ $50.1^{\circ}, \mathrm{R} 1=0.0935$. Figure 6 illustrates the structure as determined. Full data can be obtained on request from the CCDC [51].

\section{(Z)-2-(4-Nitrobenzylidene)-2H-thiazolo[3,2-a]quinazoline- 1,5-dione (9e)}

Recrystallized from dioxane/DMF (1:1) mixture as yellow crystals: $(87 \%)$, m.p. $296-297^{\circ} \mathrm{C}$; IR $(\mathrm{KBr}): v / \mathrm{cm}^{-1}$ 1716, 1675 (2CO); ${ }^{1} \mathrm{H}-\mathrm{NMR}$ (DMSO- $\left.d_{6}\right): \delta=7.67(\mathrm{t}, J=$ $8.4 \mathrm{~Hz}, 1 \mathrm{H}, \mathrm{Ar}-\mathrm{H}), 7.96(\mathrm{t}, J=8.4 \mathrm{~Hz}, 1 \mathrm{H}, \mathrm{Ar}-\mathrm{H}), 8.01$ (d, $J=8.4 \mathrm{~Hz}, 2 \mathrm{H}, \mathrm{Ar}-\mathrm{H}), 8.19$ (d, $J=8.4 \mathrm{~Hz}, 1 \mathrm{H}, \mathrm{Ar}-\mathrm{H})$, $8.26(\mathrm{~s}, 1 \mathrm{H}$, olefinic $C H), 8.39(\mathrm{~d}, J=8.4 \mathrm{~Hz}, 2 \mathrm{H}, \mathrm{Ar}-\mathrm{H})$ and $8.98 \mathrm{ppm}(\mathrm{d}, J=8.0 \mathrm{~Hz}, 1 \mathrm{H}, \mathrm{Ar}-\mathrm{H}) ;{ }^{13} \mathrm{C}-\mathrm{NMR}$ $\left(\mathrm{DMSO}-d_{6}\right.$ at $\left.80^{\circ} \mathrm{C}\right): \delta=116.3,117.6,123.3,124.5$, 128.0, 128.0, 131.3, 132.8, 134.7, 136.9, 138.8, 147.8, 163.8, 163.9 and $165.1 \mathrm{ppm}$ (Ar-C and CO); MS (EI): $m / z \quad \%) 352\left(\mathrm{M}^{+}+1,19.99\right), 351\left(\mathrm{M}^{+}, 100\right)$; HRMS (EI): $m / z$ calcd. for $\mathrm{C}_{17} \mathrm{H}_{9} \mathrm{~N}_{3} \mathrm{O}_{4} \mathrm{~S}\left(\mathrm{M}^{+}\right)$351.0308, found 351.0309 .

(Z)-2-(3-Nitrobenzylidene)-2H-thiazolo[3,2-a]quinazoline-1,5dione (9f)

Recrystallized from dioxane/DMF (1:2) mixture as orange crystals: $(82 \%)$, m.p. $238-239^{\circ} \mathrm{C}$; IR $(\mathrm{KBr}): v / \mathrm{cm}^{-1}$ 1728, 1673 (2CO); ${ }^{1} \mathrm{H}-\mathrm{NMR}$ (DMSO- $\left.d_{6}\right): \delta=7.66(\mathrm{t}, J=$ $7.8 \mathrm{~Hz}, 1 \mathrm{H}, \mathrm{Ar}-\mathrm{H}), 7.89$ (t, $J=7.8 \mathrm{~Hz}, 1 \mathrm{H}, \mathrm{Ar}-\mathrm{H}), 7.92(\mathrm{t}$, $J=8.4 \mathrm{~Hz}, 1 \mathrm{H}, \mathrm{Ar}-\mathrm{H}), 8.13(\mathrm{~d}, J=7.8 \mathrm{~Hz}, 1 \mathrm{H}, \mathrm{Ar}-\mathrm{H})$, $8.19(\mathrm{~d}, J=8.4 \mathrm{~Hz}, 1 \mathrm{H}, \mathrm{Ar}-\mathrm{H}), 8.29(\mathrm{~s}, 1 \mathrm{H}$, olefinic $C H)$, $8.33(\mathrm{~d}, J=8.4 \mathrm{~Hz}, 1 \mathrm{H}, \mathrm{Ar}-\mathrm{H}), 8.53(\mathrm{~s}, 1 \mathrm{H}, \mathrm{Ar}-\mathrm{H})$, and $8.96 \mathrm{ppm}(\mathrm{d}, J=7.8 \mathrm{~Hz}, 1 \mathrm{H}, \mathrm{Ar}-\mathrm{H}) ;{ }^{13} \mathrm{C}-\mathrm{NMR}$ (DMSO$d_{6}$ at $80^{\circ} \mathrm{C}$ ): $\delta=116.3,117.9,122.1,124.7,124.9,127.9$, $128.0,131.0,133.4,134.4,134.5,135.3,136.9,148.6$, 163.3, 163.7 and $164.9 \mathrm{ppm}$ (Ar-C and CO); MS (EI): $\mathrm{m} /$ $z$ (\%) $352\left(\mathrm{M}^{+}+1,17.78\right), 351\left(\mathrm{M}^{+}, 100\right)$; HRMS (EI): $m / z$ calcd. for $\mathrm{C}_{17} \mathrm{H}_{9} \mathrm{~N}_{3} \mathrm{O}_{4} \mathrm{~S}\left(\mathrm{M}^{+}\right)$351.0308, found 351.0308. Crystal data: $\mathrm{C}_{17} \mathrm{H}_{9} \mathrm{~N}_{3} \mathrm{O}_{4} \mathrm{~S}, \mathrm{M}=351.34$, monoclinic, $\mathrm{a}=$ 8.2228(6) $\AA, \mathrm{b}=27.550(2) \AA, \mathrm{c}=6.7703(5) \AA, \mathrm{V}=$ 1449.3(2) $\AA^{3}, \alpha=\gamma=90.00^{\circ}, \beta=109.096(7)^{\circ}$, space group: P21/c (\#14), $Z=4, D_{\text {calc }}=1.610 \mathrm{Mg} \mathrm{cm}^{-3}$, No. of reflections measured $9355,2 \theta_{\max }=52.7^{\circ}, \mathrm{R} 1=$ 0.0482 . Figure 7 illustrates the structure as determined. Full data can be obtained on request from the CCDC [52].

\section{(Z)-2-[4-(Dimethylamino)benzylidene]-2H-thiazolo[3,2-a]-} quinazoline-1,5-dione $(9 \mathrm{~g})$

Recrystallized from DMF as deep orange crystals: (78\%), m.p. 289-290 ${ }^{\circ} \mathrm{C}$; IR (KBr): $v / \mathrm{cm}^{-1} 1707,1671(2 \mathrm{CO}) ;{ }^{1} \mathrm{H}-$ NMR (DMSO- $\left.d_{6}\right): \delta=3.08\left(\mathrm{~s}, 6 \mathrm{H}, 2 \mathrm{CH}_{3}\right), 6.99(\mathrm{~d}, J=9.0$ $\mathrm{Hz}, 2 \mathrm{H}, \mathrm{Ar}-\mathrm{H}), 7.58$ (d, $J=9.0 \mathrm{~Hz}, 2 \mathrm{H}, \mathrm{Ar}-\mathrm{H}), 7.63$ (t, $J=$ 8.4 Hz, $1 \mathrm{H}, \mathrm{Ar}-\mathrm{H}), 7.88$ (t, $J=8.4 \mathrm{~Hz}, 1 \mathrm{H}, \mathrm{Ar}-\mathrm{H}), 8.02$ (s, $1 \mathrm{H}$, olefinic $C H), 8.18(\mathrm{~d}, J=8.4 \mathrm{~Hz}, 1 \mathrm{H}, \mathrm{Ar}-\mathrm{H})$ and $9.04 \mathrm{ppm}(\mathrm{d}, J=8.4 \mathrm{~Hz}, 1 \mathrm{H}, \mathrm{Ar}-\mathrm{H}) ;{ }^{13} \mathrm{C}-\mathrm{NMR}$ (DMSO- $d_{6}$ at $\left.80^{\circ} \mathrm{C}\right): \delta=66.4\left(2 \mathrm{CH}_{3}\right), 110.0,112.3,116.3,118.1$, $119.8,127.5,127.8,132.9,133.9,137.2,137.5,152.4,163.8$, 164.3 and $165.1 \mathrm{ppm}$ (Ar-C and CO); MS (EI): $\mathrm{m} / z$ (\%) $350\left(\mathrm{M}^{+}+1,23.97\right), 349\left(\mathrm{M}^{+}, 100\right)$; HRMS (EI): $\mathrm{m} / z$ calcd. for $\mathrm{C}_{19} \mathrm{H}_{15} \mathrm{~N}_{3} \mathrm{O}_{2} \mathrm{~S}\left(\mathrm{M}^{+}\right)$349.0879, found 349.0878.

\section{(Z)-2-(Thiophen-2-ylmethylene)-2H-thiazolo[3,2-a]- quinazoline-1,5-dione (9h)}

Recrystallized from DMF as pale orange crystals: (76\%), m.p. 269-270 ${ }^{\circ} \mathrm{C}$; IR (KBr): $v / \mathrm{cm}^{-1} 1705,1671$ (2CO); ${ }^{1} \mathrm{H}-\mathrm{NMR}\left(\mathrm{DMSO}-d_{6}\right): \delta=7.36(\mathrm{t}, J=6.6 \mathrm{~Hz}, 1 \mathrm{H}, \mathrm{Ar}-\mathrm{H})$, $7.65(\mathrm{t}, J=8.4 \mathrm{~Hz}, 1 \mathrm{H}, \operatorname{Ar}-\mathrm{H}), 7.79(\mathrm{~d}, J=6.6 \mathrm{~Hz}, 1 \mathrm{H}$, Ar-H), $7.90(\mathrm{t}, J=8.4 \mathrm{~Hz}, 1 \mathrm{H}, \mathrm{Ar}-\mathrm{H}), 8.09(\mathrm{~d}, J=6.6 \mathrm{~Hz}$, $1 \mathrm{H}, \mathrm{Ar}-\mathrm{H}), 8.18(\mathrm{~d}, J=8.4 \mathrm{~Hz}, 1 \mathrm{H}, \mathrm{Ar}-\mathrm{H}), 8.39(\mathrm{~s}, 1 \mathrm{H}$, olefinic $\mathrm{CH}$ ) and $8.99 \mathrm{ppm}(\mathrm{d}, J=8.4 \mathrm{~Hz}, 1 \mathrm{H}, \mathrm{Ar}-\mathrm{H})$; ${ }^{13} \mathrm{C}-\mathrm{NMR}$ (DMSO- $d_{6}$ at $80^{\circ} \mathrm{C}$ ) $: \delta=116.1,116.3,117.9$, 127.7, 127.9, 129.0, 129.3, 134.2, 134.2, 135.5, 137.1, 137.1, 163.3, 163.8 and $165.0 \mathrm{ppm}$ (Ar-C and CO); MS (EI): $m / z$ (\%) $313\left(\mathrm{M}^{+}+1,19.27\right), 312\left(\mathrm{M}^{+}, 100\right)$; HRMS (EI): $m / z$ calcd. for $\mathrm{C}_{15} \mathrm{H}_{8} \mathrm{~N}_{2} \mathrm{O}_{2} \mathrm{~S}_{2}\left(\mathrm{M}^{+}\right)$312.0021, found 312.0021. Crystal data: $\mathrm{C}_{15} \mathrm{H}_{8} \mathrm{~N}_{2} \mathrm{O}_{2} \mathrm{~S}_{2}, \quad \mathrm{M}=312.37$, monoclinic, $\mathrm{a}=3.89160(10) \AA, \mathrm{b}=27.5592(8) \AA, \mathrm{c}=$ 12.1569(3) $\AA, V=1293.43(6) \AA^{3}, \alpha=\gamma=90.00^{\circ}, \beta=$ 97.2380(10) $)^{\circ}$, space group: $\mathrm{P} 121 / \mathrm{n} 1, \mathrm{Z}=4, \mathrm{D}_{\text {calc }}=$ $1.604 \mathrm{Mg} \mathrm{cm}^{-3}$, No. of reflections measured 8288, $2 \theta_{\max }=66.65^{\circ}, \mathrm{R} 1=0.0320$. Figure 8 illustrates the structure as determined. Full data can be obtained on request from the CCDC [53].

\section{(Z)-2-(2,4-Dimethoxybenzylidene)-2H-thiazolo[3,2-a]- quinazoline-1,5-dione (9i)}

Recrystallized from DMF as yellow crystals, yield: (74\%), m.p. 254-255 ${ }^{\circ} \mathrm{C}$; IR (KBr): $v / \mathrm{cm}^{-1} 1712,1685$ (2CO); ${ }^{1} \mathrm{H}-\mathrm{NMR}\left(\mathrm{DMSO}-d_{6}\right): \delta=3.90\left(\mathrm{~s}, 3 \mathrm{H}, \mathrm{OCH}_{3}\right), 3.98$ $\left(\mathrm{s}, 3 \mathrm{H}, \mathrm{OCH}_{3}\right), 6.76$ (d, $\left.J=9.0 \mathrm{~Hz}, 2 \mathrm{H}, \mathrm{Ar}-\mathrm{H}\right), 7.54-7.64$ (m, 2H, Ar-H), 7.90 (s, 1H, Ar-H), 8.18 (d, $J=8.4 \mathrm{~Hz}$, $1 \mathrm{H}, \mathrm{Ar}-\mathrm{H}), 8.25(\mathrm{~s}, 1 \mathrm{H}$, olefinic $\mathrm{CH})$ and $9.00 \mathrm{ppm}(\mathrm{d}, J$ $=8.4 \mathrm{~Hz}, 1 \mathrm{H}, \mathrm{Ar}-\mathrm{H}) ; \mathrm{MS}(\mathrm{EI}): \mathrm{m} / z$ (\%) $367\left(\mathrm{M}^{+}+1\right.$, 24.15), $366\left(\mathrm{M}^{+}, 100\right)$; HRMS (EI): $m / z$ calcd. for $\mathrm{C}_{19} \mathrm{H}_{14} \mathrm{~N}_{2} \mathrm{O}_{4} \mathrm{~S}\left(\mathrm{M}^{+}\right)$366.0668, found 336.0668.

\section{(Z)-Methyl-2-[5-(4-nitrobenzylidene)-2-imino-4-oxothi-} azolidin-3-yl]benzoate (D $\left.\left[\mathrm{Ar}=\mathrm{P}-\mathrm{NO}_{2} \mathrm{C}_{6} \mathrm{H}_{4}\right]\right)$

A mixture of methyl-2-(2-thiocyanatoacetamido)benzoate (4) (1.25 g, $5 \mathrm{mmol})$ and 4-nitrobenzylidene 
malononitrile $(1.0 \mathrm{~g}, 5 \mathrm{mmol})$ in ethanol $(25 \mathrm{~mL})$ containing DABCO $(0.11 \mathrm{~g}, 10 \mathrm{~mol} \%)$ were stirred at reflux for just dissolving the reaction mixture and the product began to separated from the reaction approximately after $2 \mathrm{~min}$. Then, the reaction mixture was filtered off while it hot, the precipitate is $\mathbf{9}$ and the filtrate containing the intermediate $\mathbf{D}$ which allowed to cooled to room temperature. The precipitate which formed was filtered off and washed with water and recrystallized from dioxane as pale yellow crystals: m.p. $282-283^{\circ} \mathrm{C}$; IR (KBr): $v / \mathrm{cm}^{-1} 3267(\mathrm{NH}), 1717,1705$ (2CO); ${ }^{1} \mathrm{H}-\mathrm{NMR}$ $\left(\mathrm{DMSO}-d_{6}\right): \delta=3.74\left(\mathrm{~s}, 3 \mathrm{H}, \mathrm{CH}_{3}\right), 7.55(\mathrm{~d}, J=8.0 \mathrm{~Hz}$, $1 \mathrm{H}, \operatorname{Ar}-\mathrm{H}), 7.66(\mathrm{t}, J=8.0 \mathrm{~Hz}, 1 \mathrm{H}, \mathrm{Ar}-\mathrm{H}), 7.80-7.83$ $(\mathrm{m}, 2 \mathrm{H}, 1 \mathrm{Ar}-\mathrm{H}$ and olefinic $\mathrm{CH}), 7.88(\mathrm{~d}, J=8.4 \mathrm{~Hz}, 2 \mathrm{H}$, Ar- $-\mathrm{H}), 8.07(\mathrm{~d}, J=8.0 \mathrm{~Hz}, 1 \mathrm{H}, \mathrm{Ar}-\mathrm{H}), 8.40(\mathrm{~d}, J=8.4$ $\mathrm{Hz}, 2 \mathrm{H}, \mathrm{Ar}-\mathrm{H})$ and $9.97 \mathrm{ppm}(\mathrm{s}, 1 \mathrm{H}, \mathrm{NH}) ;{ }^{13} \mathrm{C}-\mathrm{NMR}$ $\left(\mathrm{DMSO}-d_{6}\right.$ at $\left.80^{\circ} \mathrm{C}\right): \delta=66.3\left(\mathrm{CH}_{3}\right), 124.3,125.8,127.8$, $127.8,129.5,130.6,131.0,131.1,133.6,134.5,139.9$, 147.1, 151.9, 164.5 and $165.3 \mathrm{ppm}$ (Ar-C and CO); MS (EI): $m / z(\%) 384\left(\mathrm{M}^{+}+1,16.25\right), 383\left(\mathrm{M}^{+}, 4.95\right) ; \mathrm{HRMS}$ (EI): $m / z$ calcd. for $\mathrm{C}_{18} \mathrm{H}_{13} \mathrm{~N}_{3} \mathrm{O}_{5} \mathrm{~S}\left(\mathrm{M}^{+}\right)$383.0570, found 383.0569 .

Ethyl-2-[(4-oxo-3,4,-dihydroquinazolin-2-yl)thio]acetate (10) A solution of methyl-2-(2-thiocyanatoacetamido)benzoate $(4)(2.50 \mathrm{~g}, 10 \mathrm{mmol})$ in ethanol $(40 \mathrm{~mL})$ containing $\operatorname{DABCO}(0.11 \mathrm{~g}, 10 \mathrm{~mol} \%)$, was refluxed for $3 \mathrm{~h}$ and allowed to cool. The formed precipitate was filtered off, washed with water and then recrystallised from $\mathrm{EtOH}$ as white crystals, yield: (99\%) \{lit. [45] (72\%)\}, m.p. 184- $185^{\circ} \mathrm{C}$ \{lit. [45], mp $\left.179-180^{\circ} \mathrm{C}(\mathrm{MeOH})\right\}$; IR (KBr): $v / \mathrm{cm}^{-1} 3167(\mathrm{NH}), 1728,1697$ (2CO); ${ }^{1} \mathrm{H}-\mathrm{NMR}$ (DMSO$\left.d_{6}\right): \delta=1.20\left(\mathrm{t}, 3 \mathrm{H}, J=7.2 \mathrm{~Hz}, \mathrm{CH}_{3} \mathrm{CH}_{2}\right), 4.08(\mathrm{~s}, 2 \mathrm{H}$, $\mathrm{CH}_{2}$ ), 4.14 (q, $\left.2 \mathrm{H}, J=7.2 \mathrm{~Hz}, \mathrm{CH}_{3} \mathrm{CH}_{2}\right), 7.40-7.44$ $(\mathrm{m}, 2 \mathrm{H}, \mathrm{Ar}-\mathrm{H}), 7.75(\mathrm{t}, J=8.0 \mathrm{~Hz}, 1 \mathrm{H}, \mathrm{Ar}-\mathrm{H}), 8.03$ $(\mathrm{d}, J=8.0 \mathrm{~Hz}, 1 \mathrm{H}, \mathrm{Ar}-\mathrm{H})$ and $12.71 \mathrm{ppm}(\mathrm{s}, 1 \mathrm{H}, \mathrm{NH})$; ${ }^{13} \mathrm{C}$-NMR (DMSO- $\left.d_{6}\right): \delta=14.1\left(\mathrm{CH}_{3}\right), 32.3\left(\mathrm{CH}_{2}\right), 61.1$ $\left(\mathrm{CH}_{2}\right), 119.8,125.8,125.9,126.1,134.3,148.1,154.7$, 161.1 and $168.4 \mathrm{ppm}$ (Ar-C and CO); MS (EI): $\mathrm{m} / z$ (\%) $265\left(\mathrm{M}^{+}+1,14.25\right), 264\left(\mathrm{M}^{+}, 87.44\right)$; HRMS (EI): $\mathrm{m} / z$ calcd. for $\mathrm{C}_{12} \mathrm{H}_{12} \mathrm{~N}_{2} \mathrm{O}_{3} \mathrm{~S}\left(\mathrm{M}^{+}\right)$264.0563, found 264.0563. Crystal data: $\mathrm{C}_{12} \mathrm{H}_{12} \mathrm{~N}_{2} \mathrm{O}_{3} \mathrm{~S}, \mathrm{M}=264.31$, orthorhombic, $\mathrm{a}=10.1158(2) \AA, \mathrm{b}=7.6750(2) \AA, \mathrm{c}=31.3650(6) \AA, \mathrm{V}=$ 2435.14(9) $\AA^{3}, \alpha=\beta=\gamma=90.0^{\circ}$, space group: P b c a, $\mathrm{Z}=8, \mathrm{D}_{\text {calc }}=1.442 \mathrm{Mg} \mathrm{cm}^{-3,}$ No. of reflections measured $8543, \theta_{\max }=66.55^{\circ}, \mathrm{R} 1=0.034$. Figure 9 illustrates the structure as determined. Full data can be obtained on request from the CCDC [54].

\section{General procedure for the reaction of 10 with aromatic aldehydes}

A mixture of ethyl-2-[(4-oxo-3,4,-dihydroquinazolin-2yl)thio]acetate (10) $(1.32 \mathrm{~g}, 5 \mathrm{mmol})$ and the appropriate arylaldehyde $(5 \mathrm{mmol})$ in acetic acid $(25 \mathrm{~mL})$ containing anhydrous sodium acetate $(0.84 \mathrm{~g}, 10 \mathrm{mmol})$ was refluxed for $5 \mathrm{~h}$. The reaction mixture was then cooled to room temperature. The precipitate which formed was filtered off and washed with water and the resulting crude product was purified by recrystallization from the appropriate solvent to afford a mixture from $\mathbf{9}$ and $\mathbf{1 1}$ in ratio $1: 1$ as illustrated from the ${ }^{1} \mathrm{H}-\mathrm{NMR}$ spectra. We cannot separate the mixtures by crystallization or by long column chromatography due to the difficult solubility of the mixtures so the reported spectral data are for both products (cf. Additional file 1).

\section{(Z)-2-(4-Methylbenzylidene)-2H-thiazolo[2,3-b]quinazoline- 3,5-dione (11a) and $9 b$}

Recrystallized from dioxane as pale yellow crystals, yield: $\left(38 \%\right.$ 9b + 38\% 11a), m.p. (for mixture) $218-220^{\circ} \mathrm{C}$; IR (KBr): $v / \mathrm{cm}^{-1} 1766,1727,1704,1678$ (4CO); ${ }^{1} \mathrm{H}-\mathrm{NMR}$ (DMSO- $\left.d_{6}\right): \delta=2.37\left(\mathrm{~s}, 6 \mathrm{H}, 2 \mathrm{CH}_{3}\right), 7.38-7.41(\mathrm{~m}, 4 \mathrm{H}$, Ar-H), 7.54 (t, $J=7.6 \mathrm{~Hz}, 1 \mathrm{H}, \mathrm{Ar}-\mathrm{H}), 7.58-7.67(\mathrm{~m}, 6 \mathrm{H}$, $\operatorname{Ar}-\mathrm{H}), 7.86(\mathrm{t}, J=7.6 \mathrm{~Hz}, 1 \mathrm{H}, \mathrm{Ar}-\mathrm{H}), 7.93(\mathrm{t}, J=8.0 \mathrm{~Hz}$, $1 \mathrm{H}, \mathrm{Ar}-\mathrm{H}), 7.96(\mathrm{~s}, 1 \mathrm{H}$, olefinic $\mathrm{CH}$ for $11 \mathrm{a}), 8.12(\mathrm{~s}, 1 \mathrm{H}$, olefinic $\mathrm{CH}$ for $9 \mathrm{~b}), 8.17(\mathrm{t}, J=7.6 \mathrm{~Hz}, 2 \mathrm{H}, \mathrm{Ar}-\mathrm{H})$ and $8.98 \mathrm{ppm}(\mathrm{d}, J=8.0 \mathrm{~Hz}, 1 \mathrm{H}, \mathrm{Ar}-\mathrm{H}) ; \mathrm{MS}(\mathrm{EI}): \mathrm{m} / z(\%)$ $321\left(\mathrm{M}^{+}+1,21.55\right), 320\left(\mathrm{M}^{+}, 100\right)$; HRMS (EI): $\mathrm{m} / z$ calcd. for $\mathrm{C}_{18} \mathrm{H}_{12} \mathrm{~N}_{2} \mathrm{O}_{2} \mathrm{~S}\left(\mathrm{M}^{+}\right)$320.0613, found 320.0614.

\section{(Z)-2-(4-Methoxybenzylidene)-2H-thiazolo[2,3-b]- quinazoline-3,5-dione (11b) and 9c}

Recrysta- llized from dioxane as yellow crystals, yield: (35\% 9c $+35 \%$ 11b), m.p. (for mixture) 206-208 ${ }^{\circ}$; IR (KBr): $v / \mathrm{cm}^{-1} 1765,1719,1704,1683$ (2CO); ${ }^{1} \mathrm{H}-\mathrm{NMR}$ $\left(\mathrm{DMSO}-d_{6}\right): \delta=3.84\left(\mathrm{~s}, 6 \mathrm{H}, 2 \mathrm{OCH}_{3}\right), 7.12-7.15(\mathrm{~m}, 4 \mathrm{H}$, Ar-H), $7.53(\mathrm{t}, J=8.0 \mathrm{~Hz}, 1 \mathrm{H}, \mathrm{Ar}-\mathrm{H}), 7.60(\mathrm{t}, J=7.6 \mathrm{~Hz}$, $1 \mathrm{H}, \operatorname{Ar}-\mathrm{H}), 7.64-7.67(\mathrm{~m}, 3 \mathrm{H}, \mathrm{Ar}-\mathrm{H}), 7.71(\mathrm{~d}, J=8.4 \mathrm{~Hz}$, 2H, Ar-H), 7.85 (t, $J=8.0 \mathrm{~Hz}, 1 \mathrm{H}, \mathrm{Ar}-\mathrm{H}), 7.91(\mathrm{t}, J=8.4$ $\mathrm{Hz}, 1 \mathrm{H}, \mathrm{Ar}-\mathrm{H}), 7.95$ (s, $1 \mathrm{H}$, olefinic $\mathrm{CH}$ for $11 \mathrm{~b}), 8.10$ (s, $1 \mathrm{H}$, olefinic $\mathrm{CH}$ for 9c), $8.14(\mathrm{t}, J=7.6 \mathrm{~Hz}, 2 \mathrm{H}, \mathrm{Ar}-\mathrm{H})$ and $8.98 \mathrm{ppm}(\mathrm{d}, J=8.4 \mathrm{~Hz}, 1 \mathrm{H}, \mathrm{Ar}-\mathrm{H}) ; \mathrm{MS}(\mathrm{EI}): \mathrm{m} / z$ (\%) $337\left(\mathrm{M}^{+}+1,22.15\right), 336\left(\mathrm{M}^{+}, 100\right)$; HRMS (EI): $\mathrm{m} / z$ calcd. for $\mathrm{C}_{18} \mathrm{H}_{12} \mathrm{~N}_{2} \mathrm{O}_{3} \mathrm{~S}\left(\mathrm{M}^{+}\right)$336.0563, found 336.0562.

\section{(Z)-2-(4-Chlorobenzylidene)-2H-thiazolo[2,3-b]quinazoline- \\ 3,5-dione (11c) and $9 d$}

Recrystallized from dioxane as pale yellow crystals, yield: (34\% 9d + 34\% 11c), m.p. (for mixture) $250-252^{\circ} \mathrm{C}$; IR (KBr): $v / \mathrm{cm}^{-1} 1765(\mathrm{br}), 1698,1679$ (4CO); ${ }^{1} \mathrm{H}-\mathrm{NMR}$ $\left(\mathrm{DMSO}-d_{6}\right): \delta=7.52-7.58(\mathrm{~m}, 2 \mathrm{H}, \mathrm{Ar}-\mathrm{H}), 7.61-7.69$ $(\mathrm{m}, 6 \mathrm{H}, \mathrm{Ar}-\mathrm{H}), 7.74(\mathrm{~d}, J=8.4 \mathrm{~Hz}, 2 \mathrm{H}, \mathrm{Ar}-\mathrm{H}), 7.79$ (d, $J=8.8 \mathrm{~Hz}, 2 \mathrm{H}, \mathrm{Ar}-\mathrm{H}), 7.88(\mathrm{t}, J=8.0 \mathrm{~Hz}, 1 \mathrm{H}, \mathrm{Ar}-\mathrm{H})$, $7.95(\mathrm{t}, J=8.4 \mathrm{~Hz}, 1 \mathrm{H}, \mathrm{Ar}-\mathrm{H}), 8.02(\mathrm{~s}, 1 \mathrm{H}$, olefinic $\mathrm{CH}$ for11c), 8.16-8.20 (m, 3H, $2 \mathrm{Ar}-\mathrm{H}$ and olefinic $\mathrm{CH}$ for $9 \mathrm{~d}$ ) and $8.98 \mathrm{ppm}(\mathrm{d}, J=8.4 \mathrm{~Hz}, 1 \mathrm{H}, \mathrm{Ar}-\mathrm{H}) ; \mathrm{MS}(\mathrm{EI}): \mathrm{m} / z(\%)$ $342\left(\mathrm{M}^{+}+2,34.66\right), 341\left(\mathrm{M}^{+}+1,1985\right), 340\left(\mathrm{M}^{+}, 100\right)$; 
HRMS (EI): $m / z$ calcd. for $\mathrm{C}_{17} \mathrm{H}_{9} \mathrm{ClN}_{2} \mathrm{O}_{2} \mathrm{~S}\left(\mathrm{M}^{+}\right) 340.0067$, found 340.0067 .

\section{General procedure for the synthesis of compounds 12 and 13}

A solution of ethyl-2-[(4-oxo-3,4,-dihydroquinazolin-2-yl) thio]acetate (10) (2.64 g, $10 \mathrm{mmol}), N, N$-dimethylformamide dimethylacetal (DMF-DMA) (1.2 g, $10 \mathrm{mmol})$ in ethanol $(30 \mathrm{~mL})$ were stirred at reflux for $6 \mathrm{~h}$. The separated solid product obtained on standing at room temperature was collected by filtration, washed by EtOH and recrystallized from EtOH the dissolved product was identified as $\mathbf{1 2}$ and the undissolved one recrystalized from DMSO and identified as $\mathbf{1 3 .}$

\section{5-Oxo-5H-thiazolo[2,3-b]quinazoline-2-carboxylic acid ethyl ester (12)}

Yield: $43 \%$, m.p. $293-294^{\circ} \mathrm{C}$; IR (KBr): $v / \mathrm{cm}^{-1} 1727$, 1691 (2CO); ${ }^{1} \mathrm{H}-\mathrm{NMR}$ (DMSO- $\left.d_{6}\right): \delta=1.34$ (t, 3H, $J=$ $7.2 \mathrm{~Hz}, \mathrm{CH}_{3} \mathrm{CH}_{2}$ ), 4.37 (q, $2 \mathrm{H}, J=7.2 \mathrm{~Hz}, \mathrm{CH}_{3} \mathrm{CH}_{2}$ ), 7.55 (t, $J=8.0 \mathrm{~Hz}, 1 \mathrm{H}, \mathrm{Ar}-\mathrm{H}), 7.66(\mathrm{~d}, J=8.0 \mathrm{~Hz}, 1 \mathrm{H}, \mathrm{Ar}-\mathrm{H})$, $7.92(\mathrm{t}, J=8.0 \mathrm{~Hz}, 1 \mathrm{H}, \mathrm{Ar}-\mathrm{H}), 8.25(\mathrm{~d}, J=8.0 \mathrm{~Hz}, 1 \mathrm{H}$, $\mathrm{Ar}-\mathrm{H})$ and $8.44 \mathrm{ppm}(\mathrm{s}, 1 \mathrm{H}$, thiazole $H 3) ; \mathrm{m} / z$ (\%) 275 $\left(\mathrm{M}^{+}+1,16.46\right), 274\left(\mathrm{M}^{+}, 100\right)$; HRMS (EI): $\mathrm{m} / z$ calcd. for $\mathrm{C}_{13} \mathrm{H}_{10} \mathrm{~N}_{2} \mathrm{O}_{3} \mathrm{~S}\left(\mathrm{M}^{+}\right)$274.0406, found 274.0406. Crystal data: $\mathrm{C}_{13} \mathrm{H}_{10} \mathrm{~N}_{2} \mathrm{O}_{3} \mathrm{~S}, \mathrm{M}=274.30$, monolinic, $\mathrm{a}=10.555$ (4) $\AA, \mathrm{b}=25.556(7) \AA, \mathrm{c}=9.115(3) \AA, \mathrm{V}=2455(2) \AA^{3}$, $\alpha=\gamma=90.00^{\circ}, \beta=93.191(7)^{\circ}$, space group: $C 2 / c$ (\#15), $\mathrm{Z}=8, \mathrm{D}_{\text {calc }}=1.484 \mathrm{Mg} \mathrm{cm}^{-3}$, No. of reflections measured $2482,2 \theta_{\max }=52.70^{\circ}, \mathrm{R} 1=0.0666$. Figure 10 illustrates the structure as determined. Full data can be obtained on request from the CCDC [55].

\section{(Z)-2-[(Dimethylamino)methylene]-2H-thiazolo[3,2-a]- quinazoline-1,5-dione (13)}

Yield: $43 \%$, m.p. $277-278^{\circ} \mathrm{C}$; IR (KBr): $v / \mathrm{cm}^{-1} 1706$, 1692 (2CO); ${ }^{1} \mathrm{H}-\mathrm{NMR}$ (DMSO-d $): \delta=3.29(\mathrm{~s}, 6 \mathrm{H}$, $\left.2 \mathrm{CH}_{3}\right), 7.61(\mathrm{t}, J=7.6 \mathrm{~Hz}, 1 \mathrm{H}, \mathrm{Ar}-\mathrm{H}), 7.85(\mathrm{t}, J=7.6 \mathrm{~Hz}$, $1 \mathrm{H}, \mathrm{Ar}-\mathrm{H}), 8.08(\mathrm{~s}, 1 \mathrm{H}$, olefinic $C H), 8.15(\mathrm{~d}, J=7.6 \mathrm{~Hz}$, $1 \mathrm{H}, \mathrm{Ar}-\mathrm{H})$ and $9.21 \mathrm{ppm}(\mathrm{d}, J=7.6 \mathrm{~Hz}, 1 \mathrm{H}, \mathrm{Ar}-\mathrm{H}) ; \mathrm{m} / z$ (\%) 274 $\left(\mathrm{M}^{+}+1,19.88\right), 273\left(\mathrm{M}^{+}, 100\right)$; HRMS (EI): $\mathrm{m} / z$ calcd. for $\mathrm{C}_{13} \mathrm{H}_{11} \mathrm{~N}_{3} \mathrm{O}_{2} \mathrm{~S}\left(\mathrm{M}^{+}\right)$273.0566, found 273.0567. Crystal data: $\mathrm{C}_{13} \mathrm{H}_{11} \mathrm{~N}_{3} \mathrm{O}_{2} \mathrm{~S}, \mathrm{M}=273.32$, monolinic, $\mathrm{a}=$ 23.074(2) $\AA, \mathrm{b}=10.1009(7) \AA, \mathrm{c}=13.896(1) \AA, \mathrm{V}=676.2$ (9) $\AA^{3}, \alpha=\gamma=90.00^{\circ}, \beta=105.058(8)^{\circ}$, space group: $C 2 / c$ (\#15), $\mathrm{Z}=8, \mathrm{D}_{\text {calc }}=1.493 \mathrm{Mg} \mathrm{cm}^{-3}$, No. of reflections measured $3557,2 \theta_{\max }=54.90^{\circ}, \mathrm{R} 1=0.0381$. Figure 11 illustrates the structure as determined. Full data can be obtained on request from the CCDC [56].

\section{2-[(4-Oxo-3,4-dihydroquinazolin-2-yl)thio]acetohydrazide (14)}

A solution of ethyl-2-[(4-oxo-3,4,-dihydroquinazolin-2yl)thio]acetate (10) $(2.64 \mathrm{~g}, 10 \mathrm{mmol})$, hydrazine hydrate $99 \%(0.75 \mathrm{~g}, 15 \mathrm{mmol})$ in absolute ethanol $(30 \mathrm{~mL})$ was stirred at reflux for $2 \mathrm{~h}$. The separated solid product from the reaction mixture was collected by filtration, washed by water and recrystallized from $\mathrm{EtOH}$ as white crystals, yield: $92 \%$, m.p. above $300^{\circ} \mathrm{C}$; IR $(\mathrm{KBr}): v / \mathrm{cm}^{-1}$ 3432, 3305, 3295, $3237\left(2 \mathrm{NH}\right.$ and $\left.\mathrm{NH}_{2}\right), 1685,1651$ $(2 \mathrm{CO}) ;{ }^{1} \mathrm{H}-\mathrm{NMR}\left(\mathrm{DMSO}-d_{6}\right): \delta=3.93\left(\mathrm{~s}, 2 \mathrm{H}, \mathrm{CH}_{2}\right), 4.34$ (br, $2 \mathrm{H}, \mathrm{NH}_{2}, \mathrm{D}_{2} \mathrm{O}$ exchangeable ), $7.43(\mathrm{t}, J=8.0 \mathrm{~Hz}$, $1 \mathrm{H}, \mathrm{Ar}-\mathrm{H}$ ), 7.53 (d, $J=8.0 \mathrm{~Hz}, 1 \mathrm{H}, \mathrm{Ar}-\mathrm{H}), 7.77$ (t, $J=8.0$ $\mathrm{Hz}, 1 \mathrm{H}, \mathrm{Ar}-\mathrm{H}), 8.03$ (d, J = 8.0 Hz, 1H, Ar-H), 9.37 (br, $1 \mathrm{H}, \mathrm{NH})$ and $12.71 \mathrm{ppm}(\mathrm{s}, 1 \mathrm{H}, \mathrm{NH}) ;{ }^{13} \mathrm{C}-\mathrm{NMR}$ $\left(\mathrm{DMSO}-d_{6}\right): \delta=32.1\left(\mathrm{CH}_{2}\right), 119.9,125.7,126.0,134.6$, $148.2,155.2,161.2,166.5$ and $171.1 \mathrm{ppm}$ (Ar-C and $\mathrm{CO})$; $\mathrm{MS}$ (EI): $m / z$ (\%) $251\left(\mathrm{M}^{+}+1,4.22\right), 250\left(\mathrm{M}^{+}\right.$, 13.89); HRMS (EI): $m / z$ calcd. for $\mathrm{C}_{10} \mathrm{H}_{10} \mathrm{~N}_{4} \mathrm{O}_{2} \mathrm{~S}\left(\mathrm{M}^{+}\right)$ 250.0518 , found 250.0519 .

\section{(E)-N'-(4-Chlorobenzylidene)-2-[(4-oxo-3,4-dihydro- quinazolin-2-yl)thio]acetohydrazide (15)}

A solution of the acetohydrazide 14 (1.25 g, $5 \mathrm{mmol})$, 4chlorobenzaldehyde $(0.70 \mathrm{~g}, 5 \mathrm{mmol})$ in ethanol $(25 \mathrm{~mL})$ containing DABCO (0.11 g, $10 \mathrm{~mol} \%)$ was stirred at reflux for $5 \mathrm{~h}$. The separated solid product obtained on standing at room temperature was collected by filtration, washed by $\mathrm{EtOH}$ and recrystallized from dioxane/DMF (1:1) mixture as white crystals, yield: 77\%, m.p. 234-235 ${ }^{\circ} \mathrm{C}$; IR (KBr): $v / \mathrm{cm}^{-1} 3438,3173$ (2NH), 1673 (br 2CO); ${ }^{1} \mathrm{H}-\mathrm{NMR}$ (DMSO- $\left.d_{6}\right): \delta=3.57\left(\mathrm{~s}, 2 \mathrm{H}, \mathrm{CH}_{2}\right), 7.40-7.52$ $(\mathrm{m}, 4 \mathrm{H}, \mathrm{Ar}-\mathrm{H}), \quad 7.72-7.76(\mathrm{~m}, 3 \mathrm{H}, \mathrm{Ar}-\mathrm{H}), \quad 8.02-8.05$ $(\mathrm{m}, 2 \mathrm{H}, 1 \mathrm{Ar}-\mathrm{H}$ and amidine $\mathrm{CH}), 11.74(\mathrm{br}, 1 \mathrm{H}, \mathrm{NH})$ and $12.70 \mathrm{ppm}(\mathrm{s}, 1 \mathrm{H}, \mathrm{NH}) ;{ }^{13} \mathrm{C}-\mathrm{NMR}$ (DMSO- $d_{6}$ ): $\delta=$ $31.8\left(\mathrm{CH}_{2}\right), 119.9,125.9,126.0,128.5,128.9,133.0$, 134.6, 142.1, 145.4, 148.2, 155.1, 161.1, 163.8 and 169.0 ppm (Ar-C and CO); MS (EI): $m / z$ (\%) $373\left(\mathrm{M}^{+}+1,6.18\right)$, $372\left(\mathrm{M}^{+}, 24.79\right)$; HRMS (EI): $m / z$ calcd. for $\mathrm{C}_{17} \mathrm{H}_{13} \mathrm{ClN}_{4} \mathrm{O}_{2} \mathrm{~S}$ $\left(\mathrm{M}^{+}\right)$372.0442, found 372.0442.

\section{$\mathrm{N}$-(4-Chlorophenyl)-2-[(4-oxo-3,4-dihydroquinazolin-2-yl)- thio]acetamide (16)}

A solution of ethyl-2-[(4-oxo-3,4,-dihydroquinazolin2-yl)thio]acetate (10) (1.32 g, $5 \mathrm{mmol})$, 4-chloroaniline $(0.70 \mathrm{~g}, 5 \mathrm{mmol})$ in acetic acid $(25 \mathrm{~mL})$ was stirred at reflux for $5 \mathrm{~h}$. The separated solid product obtained on standing at room temperature was collected by filtration, washed by EtOH and recrystallized from dioxane as white crystals, yield: $84 \%$, m.p. $276-278^{\circ} \mathrm{C}$; IR $(\mathrm{KBr}): v / \mathrm{cm}^{-1} 3406,3185(2 \mathrm{NH}), 1687$ (br 2CO); ${ }^{1} \mathrm{H}-$ NMR (DMSO- $\left.d_{6}\right): \delta=3.38\left(\mathrm{~s}, 2 \mathrm{H}, \mathrm{CH}_{2}\right), 7.25(\mathrm{t}, J=7.6$ $\mathrm{Hz}, 1 \mathrm{H}, \mathrm{Ar}-\mathrm{H}), 7.39-7.42$ (m, 3H, Ar-H), 7.67 (t, $J=7.6$ $\mathrm{Hz}, 1 \mathrm{H}, \mathrm{Ar}-\mathrm{H}), 7.78$ (d, $J=8.0 \mathrm{~Hz}, 2 \mathrm{H}, \mathrm{Ar}-\mathrm{H}), 7.98$ (d, $J$ $=7.6 \mathrm{~Hz}, 1 \mathrm{H}, \mathrm{Ar}-\mathrm{H}), 8.83(\mathrm{~s}, 1 \mathrm{H}, \mathrm{NH})$ and $10.87 \mathrm{ppm}$ $(\mathrm{s}, 1 \mathrm{H}, \mathrm{NH}) ;{ }^{13} \mathrm{C}-\mathrm{NMR}\left(\mathrm{DMSO}-d_{6}\right): \delta=24.0\left(\mathrm{CH}_{2}\right)$, $118.4,120.8,123.2,125.3,125.9,128.6,134.4,138.0,147.2$, 149.7, 161.6, 168.4 and $172.0 \mathrm{ppm}$ (Ar-C and CO); MS (EI): $m / z(\%) 346\left(\mathrm{M}^{+}+1,3.74\right), 345\left(\mathrm{M}^{+}, 11.25\right)$; HRMS 
(EI): $m / z$ calcd. for $\mathrm{C}_{16} \mathrm{H}_{12}^{35} \mathrm{ClN}_{3} \mathrm{O}_{2} \mathrm{~S}\left(\mathrm{M}^{+}\right)$345.0333, found 345.0331 .

\section{2-[(4-Amino-5-mercapto-4H-1,2,4-triazol-3-yl)methylthio]- $3 \mathrm{H}$-quinazolin-4-one (18)}

Carbon disulphide $(20 \mathrm{mmol})$ was added drop wise to an ice cold solution of potassium hydroxide $(10 \mathrm{mmol})$ in absolute alcohol $(30 \mathrm{ml})$ containing acetohydrazide $\mathbf{1 4}$ $(1.25 \mathrm{~g}, 5 \mathrm{mmol})$. The reaction mixture was stirred continuously for $24 \mathrm{~h}$ at room temperature. The precipitated potassium thiocarbamate $\mathbf{1 7}$ was filtered off, washed with chilled diethyl ether then dried and directly used for the next step without further purification. The above potassium thiocarbamate was mixed with water $(8 \mathrm{~mL})$ and hydrazine hydrate $(15 \mathrm{mmol})$ and refluxed for $4 \mathrm{~h}$. The reaction mixture turned green with evolution of hydrogen sulphide and finally it became homogeneous. The reaction mixture was cooled to room temperature and poured onto ice cold water. On acidification with acetic acid, the required triazole $\mathbf{1 8}$ was precipitated then filtered off and washed with cold water and dried. It was purified by recrystallization from EtOH/DMF (1:2) mixture to get white, crystalline solid. yield: $66 \%$, m.p. 204-205 ${ }^{\circ} \mathrm{C}$; IR $(\mathrm{KBr}): v / \mathrm{cm}^{-1} 3327,3301,3263$, $3202\left(2 \mathrm{NH}\right.$ and $\left.\mathrm{NH}_{2}\right), 1665(\mathrm{CO}) ;{ }^{1} \mathrm{H}-\mathrm{NMR}\left(\mathrm{DMSO}-d_{6}\right)$ : $\delta=4.40\left(\mathrm{~s}, 2 \mathrm{H}, \mathrm{CH}_{2}\right), 5.43\left(\mathrm{~s}, 2 \mathrm{H}, \mathrm{NH}_{2} \mathrm{D}_{2} \mathrm{O}\right.$ exchangeable ), 7.14 (t, $J=8.0 \mathrm{~Hz}, 1 \mathrm{H}, \mathrm{Ar}-\mathrm{H}), 7.32(\mathrm{~d}, J=8.0 \mathrm{~Hz}$, $1 \mathrm{H}, \mathrm{Ar}-\mathrm{H}), 7.60(\mathrm{t}, J=8.0 \mathrm{~Hz}, 1 \mathrm{H}, \mathrm{Ar}-\mathrm{H}), 7.93(\mathrm{~d}, J=8.0$ $\mathrm{Hz}, 1 \mathrm{H}, \mathrm{Ar}-\mathrm{H}), 12.85(\mathrm{~s}, 1 \mathrm{H}, \mathrm{NH})$ and $13.87 \mathrm{ppm}(\mathrm{s}, 1 \mathrm{H}$, $\mathrm{NH}) ;{ }^{13} \mathrm{C}$-NMR (DMSO- $\left.d_{6}\right): \delta=32.3\left(\mathrm{CH}_{2}\right), 116.5$, 121.1, 121.6, 124.4, 126.1, 134.0, 148.6, 152.6, 161.0 and $166.5 \mathrm{ppm}$ (Ar-C and CO); MS (EI): $m / z(\%) 307\left(\mathrm{M}^{+}+1\right.$, 21.62), $306\left(\mathrm{M}^{+}, 100\right)$; HRMS (EI): $\mathrm{m} / z$ calcd. for $\mathrm{C}_{11} \mathrm{H}_{10} \mathrm{~N}_{6} \mathrm{OS}_{2}\left(\mathrm{M}^{+}\right)$306.0352, found 306.0354.

\section{$N$-(2-Hydroxyethyl)-2-[(4-oxo-3,4-dihydroquinazolin-2-yl)- thio]acetamide (19)}

Mixture of ethyl-2-[(4-oxo-3,4,-dihydroquinazolin-2-yl) thio]acetate (10) $(2.64 \mathrm{~g}, 10 \mathrm{mmol})$, ethanolamine (1.22 $\mathrm{g}, 20 \mathrm{mmol})$ in ethanol $(25 \mathrm{~mL})$ was stirred at reflux for $4 \mathrm{~h}$. The separated solid product obtained on standing at room temperature was collected by filtration, washed by water and recrystallized from $\mathrm{EtOH}$ as colorless crystals, yield: $71 \%$, m.p. $228-229^{\circ} \mathrm{C}$; IR $(\mathrm{KBr}): v / \mathrm{cm}^{-1} 3385$, 3267, $3202(2 \mathrm{NH}$ and $\mathrm{OH}), 1689,1629(2 \mathrm{CO}) ;{ }^{1} \mathrm{H}-\mathrm{NMR}$ $\left(\right.$ DMSO- $\left.d_{6}\right): \delta=3.35\left(\mathrm{~s}, 2 \mathrm{H}, \mathrm{CH}_{2}\right), 3.40(\mathrm{q}, 2 \mathrm{H}, J=5.6$ $\left.\mathrm{Hz}, \mathrm{CH}_{2} \mathrm{OH}\right), 3.56\left(\mathrm{t}, 2 \mathrm{H}, J=5.6 \mathrm{~Hz}, \mathrm{NHCH}_{2}\right), 4.94$ (br, $1 \mathrm{H}, \mathrm{OH}, \mathrm{D}_{2} \mathrm{O}$ exchangeable), $6.37(\mathrm{~s}, 1 \mathrm{H}, \mathrm{NH})$, ), 7.10 $(\mathrm{t}, J=8.0 \mathrm{~Hz}, 1 \mathrm{H}, \mathrm{Ar}-\mathrm{H}), 7.24(\mathrm{~d}, J=8.0 \mathrm{~Hz}, 1 \mathrm{H}, \mathrm{Ar}-\mathrm{H})$, $7.56(\mathrm{t}, J=8.0 \mathrm{~Hz}, 1 \mathrm{H}, \mathrm{Ar}-\mathrm{H}), 7.88(\mathrm{~d}, J=8.0 \mathrm{~Hz}, 1 \mathrm{H}$, $\mathrm{Ar}-\mathrm{H})$ and $9.86 \mathrm{ppm}(\mathrm{s}, 1 \mathrm{H}, \mathrm{NH}) ; \mathrm{MS}(\mathrm{EI}): \mathrm{m} / z$ (\%) 280 $\left(\mathrm{M}^{+}+1,3.88\right), 279\left(\mathrm{M}^{+}, 15.09\right)$; HRMS (EI): $\mathrm{m} / z$ calcd. for $\mathrm{C}_{12} \mathrm{H}_{13} \mathrm{~N}_{3} \mathrm{O}_{3} \mathrm{~S}\left(\mathrm{M}^{+}\right)$279.0672, found 279.0671.

\section{Conclusions}

In conclusion a simple and efficient one-pot synthesis of a novel class of 2-arylidene- $2 \mathrm{H}$-thiazolo[3,2-a]quinazoline-1,5-diones 9a-i was established through DABCO catalyzed Michael type addition reaction. In addition many fused quinazoline and quinazoline derivatives were synthesized which appeared as valuable precursors in synthetic and medicinal chemistry. Moreover the X-ray single crystal technique was successfully employed in this study for structure elucidation, Z/E potential isomerism configuration determination and to determine the regioselectivity of the reactions.

\section{Additional file}

Additional file 1: ${ }^{1} \mathrm{H}-\mathrm{NMR}$ and FT-IR spectra of compounds 11a plus $9 \mathrm{~b}$ and $11 \mathrm{~b}$ plus $9 \mathrm{c}$.

\section{Competing interests}

The authors declare that they have no competing interests.

\section{Authors' contributions}

The current study is an outcome of the constructive discussion and work between $\mathrm{HB}$ and $\mathrm{HMI}$, who carried out the synthesis, purification and characterization of the compounds by the different analysis tools such as the HRMS, ${ }^{1} \mathrm{H}$ NMR, ${ }^{13} \mathrm{C}$ NMR spectral analyses and the X-ray single crystal

analysis. Both $\mathrm{HB}$ and $\mathrm{HMI}$ prepared, read and approved the final manuscript.

\section{Acknowledgments}

Support of this work was provided by the University of Kuwait through a research grant (SC03/11). The facilities of Analab/SAF supported by research grants GS01/01, GS01/05, GS01/03 and GS03/08 are gratefully acknowledged.

\section{Author details}

'Chemistry Department, Faculty of Science, Kuwait University, P.O. Box 5969, Safat 13060, Kuwait. ${ }^{2}$ Chemistry Department, Faculty of Science, Fayoum University, Fayoum 63514, A. R, Egypt.

Received: 27 February 2013 Accepted: 18 April 2013 Published: 7 May 2013

\section{References}

1. Wu J, Sun X, Li Y: DABCO: An efficient organocatalyst in the ring-opening reactions of aziridines with amines or thiols. Eur J Org Chem 2005, 20:4271-4275

2. Yamguchi K, Eto M, Higashi K, Yoshitake Y, Harano K: DABCO-triggered mild cascade reaction of electron-eficient cyclopentadienone: facile and efficient synthesis of condensed carbocycles. Tetrahedron Lett 2011, 52:6082-6085

3. Wu J-W, Li F, Zheng Y, Nie J: DABCO-mediated one-pot sequential transformation: convenient access to fluorinated $1 \mathrm{H}$-pyrazol-5(4H)-ones. Tetrahedron Lett 2012, 53:4828-4831.

4. Palomo C, Oiarbide M, Mielgo A: Unveiling reliable catalysts for the asymmetric nitroaldol (Henry) reaction. Angew Chem Int Ed 2004, 43:5442-5444.

5. Wang R, Yue L, Pan W, Zhao J-J: Direct transition metal-free C-S bond formation: synthesis of 2-aminobenzothiazole derivatives via basemediated approach. Tetrahedron Lett 2012, 53:4529-4531.

6. Abdel Gawad NM, Georgey HH, Youssef RM, El-Sayed NA: Synthesis and antitumor activity of some 2,3-disubstituted quinazolin-4(3H)-ones and 4,6-disubstituted-1,2,3,4-tetrahydro- quinazolin-2H-ones. Eur J Med Chem 2010, 45:6058-6067.

7. Chandrika PM, Yakaiah T, Rao AR, Narsaiah B, Reddy NC, Sridhar V, Rao JV: Synthesis of novel 4,6-disubstituted uinazoline derivatives, their antiinflammatory and anti-cancer activity (cytotoxic) against U937 leukemia cell lines. Eur J Med Chem 2008, 43:846-852. 
8. Al-Omary FAM, Hassan GS, El-Messery SM, El-Subbagh HI: Substituted thiazoles V. Synthesis and antitumor activity of novel thiazolo[2,3-b] quinazoline and pyrido[4,3- $d$ ]thiazolo[3,2-a]- pyrimidine analogues. Eur $J$ Med Chem 2012, 47:65-72.

9. Marvania B, Lee P-C, Chaniyara R, Dong H, Suman S, Kakadiya R, Chou T-C, Lee T-C, Shah A, Su T-L: Design, synthesis and antitumor evaluation of phenyl N-mustard-quinazoline conjugates. Bioorg Med Chem 2011 19:1987-1998.

10. Decker M, Kraus B: Design, synthesis and pharmacological evaluation of hybrid molecules out of quinazolinimines and lipoic acid lead to highly potent and selective butyrylcholinesterase inhibitors with antioxidant properties. Bioorg Med Chem 2008, 16:4252-4261.

11. El-Gazzar ABA, Youssef MM, Youssef AMS, Abu-Hashem AA, Badria FA: Design and synthesis of azolopyrimidoquinolines, pyrimidoquinazolines as anti-oxidant, anti-inflamm- atory and analgesic activities. Eur J Med Chem 2009, 44:609-624.

12. Kumar A, Sharma S, Bajaj AK, Sharma S, Panwar H, Singh T, Srivastava VK: Some new 2,3,6-trisubstituted quinazolinones as potent antiinflammatory, analgesic and COX-II inhibitors. Bioorg Med Chem 2003, 11:5293-5299.

13. Aly MM, Mohamed YA, El-Bayouki KM, Basyouni WM, Abbas SY: Synthesis of some new 4(3H)-quinazolinone-2-carboxaldehyde thiosemicarbazones and their metal complexes and a study on their anticonvulsant, analgesic, cytotoxic and antimicrobial activities. Eur J Med Chem 2010, 45:3365-3373.

14. Pandeya SN, Sriram D, Nath G, Clercq ED: Synthesis antibacterial antifungal and anti-HIV evaluation of Schiff and Mannich bases of isatin derivatives with 3-amino-2-methylmercapto quinazolin-4(3H)-one. Pharm Acta Helv 1999, 74:11-17

15. Selvam P, Girija K, Nagarajan G, De Clerco E: Synthesis, antibacterial, and anti-HIV activities of 3-[5-amino-6-(2,3-dichlorophenyl)-[1,2,4]triazin-3-yl]6,8-dibromo-2-substituted-3H-quin- azolin-4-one. Indian J Pharm Sci 2005, 67:484-487

16. Ghorab MM, Abdel-Gawad SM, El-Gaby MSA: Synthesis and evaluation of some new fluorinated hydroquinazoline derivatives as antifungal agents. II Farmaco 2000, 55:249-255.

17. Cakici M, Catir M, Karabuga S, Kilic H, Ulukanli S, Gulluce M, Orhan F: Synthesis and biological evaluation of (S)-4-aminoquinazoline alcohols. Tetrahedron-Asymmetr 2010, 21:2027-2031.

18. Alagarsamy $V$, Pathak US: Synthesis and antihypertensive activity of novel 3-benzyl-2-substituted-3H-[1,2,4]triazolo[5,1-b]quinazolin-9-ones. Bioorg Med Chem 2007, 15:3457-3462.

19. Agarwal KC, Sharma V, Shakya N, Gupta S: Design and synthesis of novel substituted quinazoline derivatives as antileishmanial agents. Bioorg Med Chem Lett 2009, 19:5474-5477.

20. Kashawa SK, Kashawa V, Mishra P, Jain NK, Stables JP: Synthesis, anticonvulsant and CNS depressant activity of some new bioactive 1-(4substituted-phenyl)-3-(4-oxo-2- phenyl/ethyl-4H-quinazolin-3-yl)urea. Eur J Med Chem 2009, 44:4335-4343.

21. Liesen AP, Aquino TM, Carvalho CS, Lima VT, Araujo JM, Lima JG, Faria AR, Melo EJT, Alves AJ, Alves EW, Alves AQ, Goes AJS: Synthesis and evaluation of anti-Toxoplasma gondii and antimicrobial activities of thiosemicarbazides, 4-thiazolidinones and 1,3,4-thiadiazoles. Eur J Med Chem 2010, 45:3685-3691.

22. Omar K, Geronikaki A, Zoumpoulakis P, Camoutsis C, Sokovic M, Ciric A, Glamoclija J: Novel 4-thiazolidinone derivatives as potential antifungal and antibacterial drugs. Bioorg Med Chem 2010, 18:426-432.

23. Liu X, Zheng C, Sun L, Liu X, Piao H: Synthesis of new chalcone derivatives bearing 2,4-thiazolidinedione and benzoic acid moieties as potential anti-bacterial agents. Eur J Med Chem 2011, 46:3469-3473.

24. Upadhyay A, Srivastava SK, Srivastava SD: Conventional and microwave assisted synthesis of some new N-[(4-oxo-2-substituted aryl-1,3thiazolidine)acetamidyl]-5-nitroindazoles and its antimicrobial activity. Eur J Med Chem 2010, 45:3541-3548.

25. Vintonyak VK, Warburg K, Kruse H, Grimme S, Hubel K, Rauh D, Waldmann $\mathrm{H}$ : Indentification of thiazolidinones spiro-fused to indolin-2-ones as potent and selective inhibitors of the mycobacterium tuberculosis protein tyrosine phosphatase B. Angew Chem 2010, 122:6038-6041.

26. Wang S, Zhao Y, Zhang G, LV Y, Zhang N, Gong P: Design, synthesis and biological evaluation of novel 4-thiazolidinones containing indolin-2-one moiety as potential antitumor agent. Eur J Med Chem 2011, 46:3509-3518.
27. Lv P, Zhou C, Chen J, Liu P, Wang K, Mao W, Li H, Yang Y, Xiong J, Zhu H: Design, synthesis and biological evaluation of thiazolidinone derivatives as potential EGFR and HER-2 kinase inhibitors. Bioorg Med Chem 2010, 18:314-319

28. Havrylyuk D, Mosula L, Zimenkovsky B, Vasylenko O, Gzella A, Lesyk R: Synthesis and anticancer activity evaluation of 4-thiazolidinones containing benzothiazole moiety. Eur J Med Chem 2010, 45:5012-5021.

29. Maccari R, Corso AD, Giglio M, Moschini R, Mura U, Ottana R: In vitro evaluation of 5-arylidene-2-thioxo-4-thiazolidinones active as aldose reductase inhibitors. Bioorg Med Chem Lett 2011, 21:200-203.

30. Rawal RK, Tripathi R, Katti SB, Pannecouque C, De Clercq E: Design and synthesis of 2-(2,6-dibromophenyl)-3-heteroaryl-1,3-thiazolidin-4-ones as anti-HIV agents. Eur J Med Chem 2008, 43:2800-2806.

31. Mobinikhaledi A, Foroughifar N, Ebrahimi S, Rahimi F, Zandi F: Synthesis of some novel 2-aryl- idene thiazoloquinazolinone derivatives via one-pot, threecomponent reaction. Phosphorus Sulfur Silicon Relat Elem 2011, 186:457-463.

32. Feng $Y$, Ding $X$, Chen T, Chen L, Liu F, Jia X, Luo X, Shen $X$, Chen $K$, Jiang $H$, Wang $H$, Liu $H$, Liu D: Design, synthesis, and interaction study of quinazoline-2 $(1 H)$-thione derivatives as novel potential $\mathrm{BCl}-\mathrm{xL}$ inhibitors. J Med Chem 2010, 53:3465-3479.

33. Bouillon I, Krchnak V: Efficient solid-phase synthesis of 3-substituted-5Oxo-5H-thiazolo[2,3-b]quinazoline-8-carboxamides under mild conditions with two diversity positions. J Comb Chem 2007, 9:912-915.

34. Loge C, Testard A, Thierry V, Lozach O, Blairvacq M, Robert J-M, Meijer L, Besson T: Novel 9-oxo-thiazolo[5,4-f]quinazoline-2-carbonitrile derivatives as dual cyclin-dependent kinase 1 (CDK1)/glycogen synthase kinase-3 (GSK-3) inhibitors: synthesis, biological evaluation and molecular modeling studies. Eur J Med Chem 2008, 43:1469-1477.

35. Testard A, Loge C, Leger B, Robert J-M, Lozach O, Blairvacq M, Meijer L, Thiery $V$, Besson $T$ : Thiazolo[5,4-f]quinazolin-9-ones, inhibitors of glycogen synthase kinase-3. Bioorg Med Chem Lett 2006, 16:3419-3423.

36. Mclntyre NA, Mclnnes C, Griffiths G, Barnett AL, Kontopidis G, Slawin AMZ, Jackson W, Thomas M, Zheleva DI, Wang S, Blake DG, Westwood NJ, Fischer PM: Design, synthesis, and evaluation of 2-methyl- and 2-amino- $\mathrm{N}$-aryl4,5-dihydrothiazolo[4,5-h]quinazolin-8-amines as ring-constrained 2anilino-4-(thiazol-5-yl)pyrimidine cyclin-dependent kinase inhibitors. J Med Chem 2010, 53:2136-2145

37. Mrkvicka V, Klasek A, Kimmel R, Pevee A, Kosmrlj J: Thermal reaction of $3 \mathrm{aH}, 5 \mathrm{H}$-thiazolo[5,4-c]quinoline-2,4-diones an easy pathway to 4-amino$1 \mathrm{H}$-quinoline-2-ones and novel $6 \mathrm{H}$-thiazolo[3,4-c]quinazoline-3,5-diones. ARKIVOC 2008, 14:289-302.

38. Grasso S, Micale N, Monforte A-M, Monforte P, Polimeni S, Zappala M: Synthesis and in vitro antitumour activity evaluation of 1-aryl-1 $\mathrm{H}, 3 \mathrm{H}$ thiazolo[4,3-b]quinazolines. Eur J Med Chem 2000, 35:1115-1119.

39. Bakherad M, Keivanloo A, Kalantar Z, Keley V: Regioselective syntheses of 1aryl-substituted-5H-[1,3]thiazolo[3,2-a]quinazoline-5-ones during Sonogashira coupling. Phosphorus Sulfur Silicon Relat Elem 2011, 186:464-470.

40. Jain KK, Rout SP, Pujari HK: Heterocyclic systems containing bridgehead nitrogen atom. Part LXIV. Synthesis of 7-methylthiazolo[3,2-a] quinazolines. Indian J Chem Sect B 1990, 29B:379-80.

41. Behbehani $\mathrm{H}$, Ibrahim HM, Makhseed S, Mahmoud H: Applications of 2 arylhydrazononitriles in synthesis: preparation of new indole containing 1,2,3triazole, pyrazole and pyrazolo[1,5-a]pyrimidine derivatives and evaluation of their antimicrobial activities. Eur J Med Chem 2011, 46:1813-1820.

42. Behbehani H, Ibrahim HM, Makhseed S, Elnagdi MH, Mahmoud H: 2Aminothiophenes as building blocks in heterocyclic synthesis: synthesis and antimicrobial evaluation of a new class of pyrido[1,2-a]thieno[3,2-e] pyrimidine, quinoline and pyridin-2-one derivatives. Eur J Med Chem 2012, 52:61-65.

43. Behbehani $H$, Ibrahim HM: 4-Thiazolidinones in heterocyclic synthesis: synthesis of novel enaminones, azolopyrimidines and 2-Arylimino-5arylidene-4-thiazolidinones. Molecules 2012, 17:6362-6385.

44. Vicini P, Geronikaki A, Anastasia K, Incerti M, Zani F: Synthesis and antimicrobial activity of novel 2-thiazolylimino-5-arylidene-4thiazolidinones. Bioorg Med Chem 2006, 14:3859-3864.

45. Gruner M, Rehwald M, Eckert K, Gewald K: New synthesis of 2-alkylthio-4oxo-3,4-dihydroquinazolines, 2-alkylthioquinazolines, as well as, their hetero analogues. Heterocycles 2000, 53:2363-2377.

46. Crystallographic data for 3 (ref. CCDC 916675) can be obtained on request from the director. 12 Union Road, Cambridge CB2 1EW, UK: Cambridge Crystallographic Data Center. 
47. Crystallographic data for 4 (ref. CCDC 916665) can be obtained on request from the director. 12 Union Road, Cambridge CB2 1EW, UK: Cambridge Crystallographic Data Center.

48. Cnystallographic data for 5 (ref. CCDC 916666) can be obtained on request from the director. 12 Union Road, Cambridge CB2 1EW, UK: Cambridge Crystallographic Data Center.

49. Crystallographic data for $9 a$ (ref. CCDC 916667) can be obtained on request from the director. 12 Union Road, Cambridge CB2 1EW, UK: Cambridge Crystallographic Data Center.

50. Crystallographic data for 9c (ref. CCDC 916674) can be obtained on request from the director. 12 Union Road, Cambridge CB2 1EW, UK: Cambridge Crystallographic Data Center.

51. Crystallographic data for $9 d$ (ref. CCDC 916671) can be obtained on request from the director. 12 Union Road, Cambridge CB2 1EW, UK: Cambridge Crystallographic Data Center.

52. Crystallographic data for if (ref. CCDC 916670) can be obtained on request from the director. 12 Union Road, Cambridge CB2 1EW, UK: Cambridge Crystallographic Data Center.

53. Crystallographic data for 9h (ref. CCDC 916673) can be obtained on request from the director. 12 Union Road, Cambridge CB2 1EW, UK: Cambridge Crystallographic Data Center.

54. Crystallographic data for 10 (ref. CCDC 916672) can be obtained on request from the director. 12 Union Road, Cambridge CB2 1EW, UK: Cambridge Crystallographic Data Center.

55. Crystallographic data for 12 (ref. CCDC 916668) can be obtained on request from the director. 12 Union Road, Cambridge CB2 1EW, UK: Cambridge Crystallographic Data Center.

56. Crystallographic data for 13 (ref. CCDC 916669) can be obtained on request from the director. 12 Union Road, Cambridge CB2 1EW, UK: Cambridge Crystallographic Data Center.

doi:10.1186/1752-153X-7-82

Cite this article as: Behbehani and Ibrahim: Organocatalysis in heterocyclic synthesis: DABCO as a mild and efficient catalytic system for the synthesis of a novel class of quinazoline, thiazolo [3,2-a] quinazoline and thiazolo[2,3-b]

quinazoline derivatives. Chemistry Central Journal 2013 7:82.

\section{Publish with ChemistryCentral and every scientist can read your work free of charge \\ "Open access provides opportunities to our colleagues in other parts of the globe, by allowing anyone to view the content free of charge." \\ W. Jeffery Hurst, The Hershey Company. \\ - available free of charge to the entire scientific community \\ - peer reviewed and published immediately upon acceptance \\ - cited in PubMed and archived on PubMed Central \\ - yours - you keep the copyright \\ Submit your manuscript here: \\ http://www.chemistrycentral.com/manuscript/<smiles>c1ccccc1</smiles> \\ Chemistry Central}

